\title{
A Game Theoretic Fault Detection Filter
}

Walter Il. Chung and Jason L. Speyer*

Mcchanical, Acrospace, and Nuclear Engincering Department

University of Califormia, Los Angcles

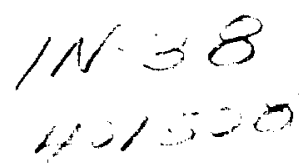

48-121 Eugr. IV

Los Angcles, CA 90095

vhchungetalus. seas.ucla.edu or speyereseas.ucla.edu

TEL: (310) 206-0898 FAX: (310) 206-2302

November 2, 1995

\begin{abstract}
The fault detertion process is modelled as a disturbance attenuation problem. The solution to this problem is found vis differential game theory, leading to an $H_{\text {ow }}$ filter which bounds the transmission of all exogenous signals save the fault to be detected. For a general class of linear systems which includes some time-varying systems, it is gown that this tranumission bound can be taken to zero by simultaneously bringing the sensor noise weighting to zero. Thus, in the limit, a complete trammission block can be achieved, making the game filter into a fault detection filter. When we specinlize this result to tine-invariant systems, it is found that the detection filter attained in the limit is identical the well known Beard-Jones Fault Detection Filler. That is, all fault inputs other than the one to be detected (the "muisanice faults") are restricted to an invariant subspace which is unobservable to a projection on the output. For time-invariant systetus, it is also sliomn that in the limit, the order of the state-space and the game filter can be

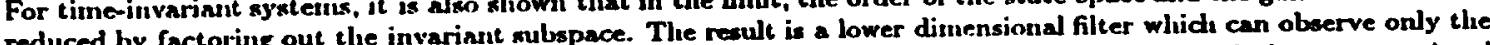
reduced by factoring out the invariant subspace. The result is a lower dimensional filter which can abserve ony che fault to be detected. A reduced-order fler can also be generated for ther demonstrates the effectiveness of the filter as a tool for fault sletection and identification.
\end{abstract}

\section{Introduction}

The need for high reliability and low maintenance in complex systems such as the proposed intelligent vehicle highway system will require self-monitoring and fault tolerance. Schemes to carry out. these tasks generally fall under the heading of health monitoring systems and without exception require a processor which uses sensor data to determine the presence and origin of failures within the system. One concept of such a processor is the fault detection filter. Often called the Beard-Jones Fault. Detection Filter after its originators, the fault detection filter is a specially designed observer which isolates the influence of each fanlt. upon the state trajectory, making the simultaneously detection and identification of failures possible.

Since its initial formulation, the fault detection filter lias undergone many reinterpretations and refinement.s. White [1] derived a detection filter design algorithm based upon eigenstruct.ure assigmment. Massoumnia [2] gave a geometric interpretation of the filtar and also derived a reduced-order fault detector based on geometric arguments in [3]. Most. recently, Douglas robustified the filter and derived a new version of the filter which bounds disturbance transmission [4], [5], and [6]

- This work was sponsenred hy NASA-Aume Research Center Comperative Agreenem No. NCC 2-374, supplement 19 and Air Force Office of Scichitific Research Cirant F4MizU-94-1-0us4 
Common to all of these incamations of the fault cletection filter is an underlying structure of independent, invariant subspaces which are matched one-to-one with a particular fault and which wholly contain the state trajectory when the system is driven solely by that. fault. Design algorithms have, thus, relied heavily on spectral methods - i.e. specifying eigenvalues or eigenvectors. Spect.ral methods lead directly to the needed filter structure, but they limit the applicability of the fault detection filter to linear, time-invariant systems and, due to their need for an accurate plant model, their robust.ness to parameter variations can be poor [7].

For these reasons, we have taken a different route to fault detection filter design. We look at the fault detection process as a disturbance attenuation problem. The filter design, as a result, comes out of a differential game in which the player is a state estimate and the adversaries are all of the exogenous signals, save the fault to be detected. To approximate the invariant subspace structure of the fault detection filter, the game is one in which the player attempts to exclude the adversaries from a specified portion of the state-space, assuming all the while that the adversaries play their best strategy. The solution to this game results in an $H_{\infty}$-type filter which bounds disturbance transmission.

Since fault detection filters block transınission, it would seem reasonable to expect that in the limiting case when the $H_{\infty}$ transmission bound is brought to zero, the game filter no longer approximates, but actually becomes a fault detection filter. We will prove that this is indeed the case. In fact, for linear time-invariant (LTI) systems, we will show that the game filter hecomes a Beard-Jones Fault Detector in the sense of [4]: faults other than the one to be detected are restricted to a subspace which is invariant and unobservable.

The filter that, we propose liere. however, is not merely an alternative algorithm for detection filter design. The method we develop has wider applicability than existing design technicues. Time invariance is not assumed in the game solution and so it can be shown that, for a class of time-varying systems, results analogous to the LTI case exist in the limit as disturbance bounds are taken to zero. With this method, it is also possible to deal with model uncertainty by treating such uncertainty as another element. in the differential game [8], [9]. Finally, we will show that with this approach the designer has the freedom to choose the extent to which the game filter behaves as an $H^{\infty}$ filter and the extent. to which it beliaves like a detection filter. This flexibility is unique to the game theoretic approach to fault detection filter design.

Section 2 covers the hasics of fault detection filter theory. We define a general fault detection filter prohlem and show how the existing theory matches our definition for LTI systems. In Section 3, we pose a disturbance at.tenuation problem which models the fault detection process for a large class of systems which inclucles some time- 
varying systems. The solution to this prohlems leads to a game theoretic filter which bounds the transmission of all exogenous inputs aside from the fault be detected. In Section 4, we analyze sufficient conditions for our bante cost. to be non-positive. This will enahle us to show the existence of the filter in the limit and analyze its st.ructure. In Section 5, we return to the LTI case and prove that the detection filter that we find in the limiting case is equivalent to the fault detection filter structures already existing in the literature. In Section 6 , we use the limiting form of the game theoretic filter to derive a reduced-order estimator for fanlt detection. These results apply to the larger class of systems. Finally, in Section 7 we go through an example which shows that the filter is an effective fault detector for finite values of the dist.urbance at.tenuation bound and in the limit.

\section{Fault Detection Filter Theory}

In this section, we introduce and define the fault detection filter problem. The existing theory for fault detection filters is strongly tied to time-invariant systems and many of the key results of this theory depend on concepts which do not. carry over into time-varying systems. Hence, we make a distinction between the existing problem and a definition that we propose for the fault deteclion problen based upon disturbance attenuation. We will shou that our definition matches the existing definition when applied to LTI systems.

\subsection{Time-Invariant Fanlt Detection Filter Theory}

Current fault detection filter theory is largely developed in [1], [2], and [4]. The geometric contiol concepts upon which much of the theory is based are developed in [10].

The systems that we consicler here are linear, time-invariant with $(C, A)$ observable:

$$
\begin{gathered}
j(t)=A x(t)+B u(t) \\
y(t)=C x(t) .
\end{gathered}
$$

Linear maps are denoted hy mathematical italics (e.g. $M$ ) and linear spaces by caligraphic text (e.b. $\mathcal{M}$ ). Hence,

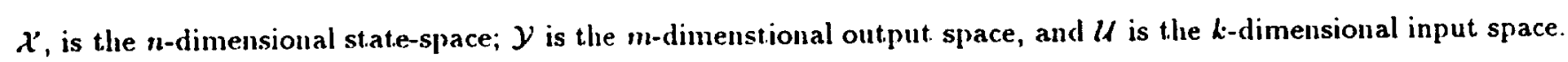
$A: x \rightarrow x, B: u-x$, and $C: x \rightarrow y$ are matrices of appropriate dimesions and are typically called the state matrix, the input matrix, and the ontput matrix respectively.

It. has been shown in the cited references that fanlts in the sensors, the actuators, and even the plant can be modelled as additive siguals in the state equation:

$$
\dot{r}=A l+B u+\sum_{j=1}^{4} F_{j} L_{j}
$$


The $F_{j}: \mathcal{M}_{j} \rightarrow l^{\prime \prime}$ s are called failue maps and are fixed, known maps which represent the directional behavior of the fault. For an actuator failure, $F_{j}$ is the corresponding column of the $B$ matrix. For sensor failures [1]. $F_{j}$ is derived from the corresponding row of the $C$ matrix via:

$$
y=C x+E_{i} \mu_{i}
$$

$E_{i}$ is an $m \times 1$ unit vector corresponding to a failure in the ith sensor. To convert this failure into a form compatible with (1), a two-column failure map [1]:

$$
F_{i}=\left[\begin{array}{ll}
f_{i} & f_{i}^{*}
\end{array}\right]
$$

is used where $f_{i}$ is any vector such that $E_{i}=C f_{i}$ and $f_{i}^{*}=A f_{i}$ - for time-invariant systems. For time-varying systems, $f_{i}^{*}=A f_{i}-\dot{f}_{i}$. Why this is so will be explained later in this section.

The $\mu_{j}$ 's are failure signals and represent the time history of the failure signal amplitude. In nominal, no-failure conditions, the $\mu_{j}$ 's are zero. Failure maps are generally assumed to be monic so that non-zero $\mu_{j}$ 's result in non-zero vectors, $F_{j} \mu_{j}$. In addition to $(C, A)$-observability, it will also be assumed that the system is output scparablc [1]:

$$
\operatorname{rank}\left[C A^{\beta_{1}} V_{1}, \ldots, C A^{\beta_{q}} V_{q}\right]=q
$$

where:

$$
V_{i}= \begin{cases}F_{i} & \text { if } i \text { is an actuator fault. } \\ f_{i} & \text { if } i \text { is a sensor fault. }\end{cases}
$$

Output separability is a check that the problem is well-posed in the sense that the failures chosen in the design set are linearly independent. $F_{i}$ is the failure map for actuat.or faults, $f_{i}$ is the vector defined by Equation 2 , and $\beta_{i}$ is the smallest integer such that $C . A^{p} \cdot V_{i} \neq 0$. We make the distinction between actuator and sensor faults. because $F_{i}$ has only one colunu for the former and two columns for the latter. If (3) is not satisfied, then the designer needs to decide which failures he needs to discard from his design set. Note, that (3) is a time-invariant result. The analog for the time-varying case would look like a grammian matrix.

Definition 2.1. The Funlf Defection Filfer Problem as defined in [4] is to fiud subspaces $\mathcal{W}_{j} \subset x$ such that:

1. Each suluspace conteains the image of one and only one of the failure maps $\left(\mathcal{F}_{j}:=\operatorname{lmage} F_{j}\right)$ :

$$
\mathcal{F}_{j} \subset \mathcal{W}_{j}
$$


2. The subspaces are ontput stpuralle:

$$
C \mathcal{W}_{j} \cap \sum_{i \neq j} c: \mathcal{W}_{i}=
$$

3. The subspaces are $(C, A)$-invariant, i.e. there exists a mal, $L: \mathcal{Y} \rightarrow \boldsymbol{x}$ such that:

$$
(A+L C) \mathcal{W}_{j} \subset \mathcal{W}_{j}
$$

Note that the output separability requirement. in (5) refers to the solution of the fault detection filter problen, whereas the output. separability specified by (3) refers to the well-posedness of the detection filter problem itself.

In [4] unobscrvability subspaces are used as the invariant subspaces for detection filter construction. Unobservability subspaces are the dual of controllablity subspaces [10] and are the unobservable subspaces, $\mathcal{T}_{j}$, of the pairs $\left(\hat{H}_{j} C, A+L C\right)$, where $\mathrm{L}$ is the observer gain from (6) and $\hat{H}_{j}$ is a natural projection such that ker $\dot{H}_{j} C=$ $\sum_{i \neq j} \mathcal{T}_{i}+\operatorname{ker} C$ (ker $C$ refers to the Kernal or Null Space of $C$ ). $\dot{H}_{j}$ can be found via:

$$
\hat{H}_{j}=I-\left(C A^{\beta ;} \dot{F}_{j}\right)\left[\left(C A^{\beta}, \hat{F}_{j}\right)^{T}\left(C A^{\beta,} \dot{F}_{j}\right)\right]^{-1}\left(C A^{\beta ;} \dot{F}_{j}\right)^{T}
$$

Again, $\beta_{j}$ is the smallest. integer such that $C A^{\beta} ; \hat{F}_{j} \neq 0$ and

$$
\hat{F}_{j}=\left[\begin{array}{llllll}
F_{1} & \ldots & F_{j-i} & F_{j+1} & \ldots & F_{q}
\end{array}\right]
$$

Roughly speaking, $\hat{H}_{j}$ blocks out the portion of the state-trajectory which lies in the other invariant subspaces.

Unobservability sulsspaces are crucial for spectral design methods because for any such subspace, $\mathcal{T}_{i}$, both the spectrum of $\left(A+L C \mid \mathcal{T}_{i}\right)$ - the restriction of $(A+L C)$ to $\mathcal{T}_{i}$ - and the spectrum of $\left(A+L C \mid \mathcal{X} / \mathcal{T}_{i}\right)$ - the restriction to the factor space - can be compleiely specified by $L^{1}$. If an invariant subspace other than an unobservability subspace is used in a detection filter design, it is possible that some of the eigenvalues of $(A+L C)$ will be fixed by the invariant zeros of $\left(C, A, F_{1}\right)$. This could be problematic if these zeros are in undesirable locations. We will briefly discuss invariant zeros and where they appear in the game filter in Section 5 so that our results can be directly compared to existing detection filter design met.hools.

When the three given conditions are realized, the result is a Luenberger Ohserver:

$$
\dot{x}=A \hat{x}+B u+L(y-C \dot{x})
$$

\footnotetext{
1 when there is only one umoliservaljility sulwpace to consider, this means that the entire spectrum of closed-loop state matrix ( $A+L C$ ) can be specified. When more lian one unul servalility involved, an extra condition called nutual detectability [1] is needed to specily all of $(A+L C)$
} 
with error dymamies governed ly $(c:=x-j)$ :

$$
\dot{c}=(A+L C) c+\sum_{j=1}^{q} F_{i} / t_{i}
$$

Equation 5 implies that. $\mu_{j}$ is restricted to an invariant subspace $\mathcal{W}_{j}$ whose image under $C$ is disjoint from all other such subspaces. Hence, by the definition of $\hat{H}_{j}$ and equations (4) - (9), the signal:

$$
z_{j}=\hat{H}_{j}(y-C \hat{x})=\hat{H}_{j} C e .
$$

will be driven solely by the fault. $\mu_{j}$. A non-zero value of $z_{j}$ should simultaneuosly detect. and identify the fault $\mu_{j}$. Given (10, we can derive the sensor failure map for the time-varying case. A sensor fault leads to $z=\hat{H}_{1} C \bar{\epsilon}$ where $\bar{e}=e+f_{i} \mu_{i}$. Differentiating $\bar{c}$, gives us an equation in the form of (9), leading to the failure map:

$$
\dot{\bar{c}}=(A+L C) \bar{e}-A f_{i} \mu_{i}+\dot{f}_{i} \mu_{i}+f_{i} \dot{\mu}_{i}=(A+L C) \bar{e}+\left[\begin{array}{ll}
f_{i} & f_{i}^{*}
\end{array}\right]\left\{\begin{array}{c}
\dot{\mu}_{i} \\
-\mu_{i}
\end{array}\right\}
$$

\subsection{A Definitiou of the Fault Detection Filter Problem based upon Disturbance Attenuation}

Let us now consider an alternative and more encompassing definition of the fault detection filter problem. Consider a linear system in which $q$ possible faults have been modelled:

$$
\begin{aligned}
& \dot{x}(t)=A(t) x(t)+B(t) u(t)+F_{1}(t) \mu_{1}(t)+\sum_{i=2}^{t} F_{i}(t) \mu_{i}(t) \\
& y(t)=C(t) x(t)+v(t) .
\end{aligned}
$$

It is desired to detect the appearance of $\mu_{1}$ (the "target fault") in the presence of sensor noise, $v$, and the possible presence of other faults $\mu_{i}, i \neq 1$ (the "nuisance faults"). Following the standard assumptions of section 2.1, we will assume that each of the $F_{i}$ 's are monic and that $(C, A)$ is an observable pair. Also, since $u$ is a known function of $t \in\left[t_{0}, t_{1}\right]$, we will drop the $B u$ term for convenience. We will also neglect to explicitly show the possible time dependence of the system matrices, though the reader should keep this possibility in mind. For convenience, we clefine:

$$
\mu_{1}=\left\{\begin{array}{c}
\mu_{2} \\
\vdots \\
\mu_{q}
\end{array}\right\}
$$

and use the definition of $\dot{F}_{i}(8)$ so that the state equation becomes:

$$
i=A x+F_{1 / \mu_{1}}+\hat{F}_{1} i_{2}
$$


The definition that we propose is hased upon disturhance attenuation. We use (10) and define the corresponding residual signal $z_{1}$ associated with $\mu_{1}$ as the output. signal. A disturbance attemuation problem would be to limit the t.ransmission of the nuisance faults and the sensor noise to this output. For a fault detection filter problem we want to block this transmission entirely.

Defuition 2.2. The Faull Detcction Filter Problem is to find an estimator such that:

$$
\frac{\left\|z_{1}\right\|^{2}}{\left\|\mu_{2}\right\|^{2}}=0
$$

and:

$$
\frac{\left\|z_{1}\right\|^{2}}{\left\|\mu_{1}\right\|^{2}} \neq 0
$$

Clearly, in the time-invariant. case, the solution to the fault detection filter problem as defined by Definition 2.1 solves the general fault detection filter problem that we have defined above. Later on, we will show that these definitions are equivalent. in the time-invariant case by showing that the solution to Definition 2.2 solves the problem defined by Definition 2.1 .

The definition that we propose is needed when we consider time-varying systems. In such cases, we cannot talk about invariant. sulsspaces and also observability becomes a trickier concept. Thus instead of defining the structure of the final filter, we must, content ourselves with merely describing its action.

Now, because we are dealing with time-varying systems, we must define our projector $\hat{H}_{1}$ appropriately:

$$
\dot{H}_{1}=I-C B_{i}\left[\left(C B_{i}\right)^{T}\left(C B_{i}\right)\right]^{-1}\left(C B_{i}\right)^{T}
$$

where:

$$
\begin{aligned}
& B_{1}=\dot{F}_{1} \\
& B_{j}=.+B_{j-1}+\dot{B}_{j-1}
\end{aligned}
$$

and $i \in \mathbb{Z}_{+}$is the smallest integer such that:

$$
C B_{i} \neq 0
$$

For time-invariant systems, (11) reduces to (7). Equations (12) to (14) come from the Golı Transformation [11]. We will use the Goh Transformation again in later sections. Il will he assumed that the system matrices $A(t), C(t), \dot{F}_{1}(t)$ 
the number of iterations of (13) needed so that (14) is satisfied is constant. over the entire interval, [to. $\left.t_{1}\right]$. That. is, the time-variations of the system do not cliange the dimensionality of the detection problem. This restricts the applicability of this analysis to a subclass of time-varying systems, but it allows us to avoid expending the effort which would be needed to deal with exceptional cases. Calling (11) a projector is a slight misnomer since it will be a time-varying mat.rix, but. by it's construction it will be such that $\operatorname{lier} \hat{H}(t)_{1}=\operatorname{Ker} C(t)+\operatorname{Im} B_{i} \forall t \in\left[t_{0}, t_{1}\right]$ which is our main concern.

\section{A Game Theoretic Filter for Fault Detection in a General Class of Systems}

\subsection{The Disturbance Attemution Problem}

We arrive at a solution to the fault detection filter problem as defined by Definition 2.2 by first solving the disturbance attenuation problem. The solution to the fault detection filter problem then comes when we take the limit of the disturbance at.tenuation solution. The results that we find here, however, are valuable in their own right. As we will see, the game filter that. we get from the distiabance attenuation problem is itself a useful filter for fault detection.

We begin by quantifying the probiem objective with a disturbance attenuation fanction, the ratio of the norm of the output to the norms of the inputs. For this problem, the function is:

$$
D_{a j}=\frac{\int_{t_{0}}^{t_{2}}\left\|\hat{H}_{1} C(x-\hat{x})\right\|_{Q_{1}}^{2} d t}{\int_{t_{1}}^{t_{3}}\left[\left\|\dot{\mu}_{2}\right\|_{M_{3}-1}^{2}+\|v\|_{V-1}^{2}+\left\|N_{1} C(x-\dot{x})\right\|_{R_{1}}^{2}\right] d t+\left\|x\left(t_{0}\right)-\bar{x}_{0}\right\|_{P_{0}^{-1}}^{2}}
$$

where $N_{1}:=I-\hat{H}_{1}$ and $M_{2}, V, R_{1}, P_{0}$ are weigliting matrices. The disturbance attenuation problem is to find an estimator so that for all adversaries $i_{2 \cdot 2}, v \in L_{2}\left[t_{1}, t_{2}\right], x(0) \in \mathbb{R}^{n}$ :

$$
D_{a f} \leq \gamma
$$

We will refer to $\gamma$ as the disturbance attenuation bound. Once again, the assumptions that we will make are: 1) $(C, A)$ is a an observable pair 2) $F_{i}, i=1 \ldots q$ is monic 3$) i$, the number of iterations of (13) needed so that. (14) is satisfied, is a const.ant.

To solve (16) we convert it. into a differential game with cost function:

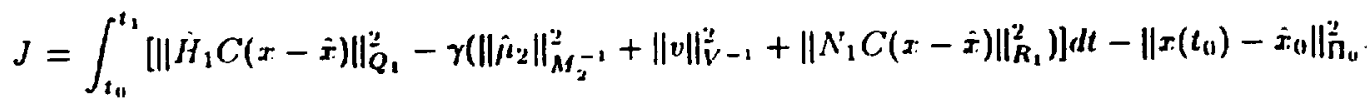

Note that $\Pi_{0}:=\gamma P_{0}^{-1}$. We want. to find:

$$
\min _{j} \max _{\nu} \max _{\dot{i}_{2}} \max _{x\left(\ell_{10}\right)} J \leq 0
$$

subject to: 


$$
\dot{r}=A x+\hat{F}_{1} \hat{\mu}_{2}
$$

An element. that is missing in our problem statement $(17),(18),(19)$ is the target fault, $\mu_{1}$. This is not an oversight. It would seem logical to include enhancing the transmission of $\mu_{1}$ as part of the game, but there is no obvious way to include such an objective in the game cost. Moreover, extremizing the cost with respect to $\mu_{1}$ leads to assumptions upon the temporal beliavior of the target fault. This can be quite detrimental to filter performance if these assumptions are wrong (which is why fault detection filters are designed without any such assumptions). Thus, since $\mu_{3}$ is not part of the differential game, we set it to zero for convenience when we work through the solution. This places the burden on the designer to make sure the set of faults that he chooses for the filter design leads to a well-posed problem. Well-posedness is discussed in Section 2 and for LTI systems is easily checked by Equation 3.

\subsection{Maximization with Respect to $x\left(t_{0}\right)$ and $i_{2}$}

We will solve our problem in two steps beginning with the subproblem:

$$
\max _{\dot{\mu}_{3}} \max _{x\left(t_{u}\right)} J \leq 0
$$

The reasoning for this particular order of the extremizations is given in [12].

We begin by converting the sensor noise term $\|v\|_{V-1}^{2}$ to the equivalent $\|y-C x\|_{V-1}^{2}$ and by appending the dynamics of the system to the cost with a Lagrange multiplier, $\lambda^{T}$ :

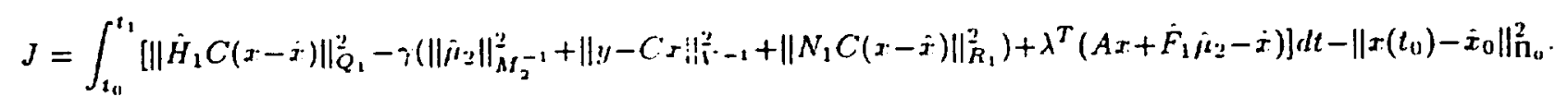

Integrate $\lambda \dot{x}$ by parts:

$$
\begin{aligned}
& J=\int_{t_{11}}^{t_{1}}\left[\left\|\dot{H}_{1} C(x-\hat{x})\right\|_{Q_{1}}^{2}-i\left(\|\dot{i}\|_{2}\left\|_{M_{2}^{-1}}^{2}+\right\| y-C x\left\|_{V^{-1}}^{2}+\right\| N_{1} C(x-\hat{x}) \|_{R_{1}}^{2}\right)\right. \\
& \left.+\lambda^{T}\left(A r+\dot{F}_{1} \hat{H}_{1}\right)+\dot{\lambda}^{T} x\right] d t-\left\|x\left(t_{01}\right)-\hat{x}_{01}\right\|_{\Pi_{10}}^{z_{0}}+\lambda\left(t_{0}\right)^{T} x\left(t_{01}\right)-\lambda\left(t_{1}\right)^{T} x\left(t_{1}\right)
\end{aligned}
$$

and then take the variation of $(21)$ with respect to $\hat{i}_{2}$, and $x\left(t_{11}\right)$ :

$$
\begin{aligned}
& \delta J=\int_{1_{11}}^{t_{1}}\left\{\left[(x-\dot{x})^{T} C^{T} \dot{H}_{1}^{T} Q_{1} \hat{H}_{1} C+\gamma(y-C x)^{T} V^{-1} C-\gamma(x-\dot{x})^{T} C^{T} N_{1}^{T} R_{1} N_{1} C+\dot{\lambda}^{T}+\lambda^{T} A\right] \delta x\right. \\
& \left.+\left[-\gamma \mu_{1}^{T} M_{2}^{-1}+\lambda^{T} \dot{F}_{1}\right] \delta_{1 \cdot 1}\right] d t-\left[\left(x\left(t_{11}\right)-\dot{x}_{(1)}\right)^{T} \Pi_{01}+\lambda\left(t_{11}\right)^{T}\right] \delta x\left(t_{0}\right)-\lambda\left(t_{1}\right)^{T} \delta x\left(t_{1}\right) .
\end{aligned}
$$


The above implies that first-order necessary conditions for $J$ to be maximized are:

$$
\begin{aligned}
\hat{H}_{2} & =\frac{1}{\gamma} M_{2} \hat{F}_{1}^{T} \lambda \\
-\dot{\lambda} & =A^{T} \lambda+C^{T}\left(\hat{H}_{1}^{T} Q_{1} \hat{H}_{1}^{T}-\gamma N_{1}^{T} R_{1} N_{1}\right) C(x-\hat{x})+\gamma C^{T} V^{-1}(y-C x) \\
\lambda\left(t_{1}\right) & =0 \\
\lambda\left(t_{0}\right) & =\Pi_{0}\left[x \cdot\left(t_{0}\right)-\hat{x}_{0}\right]
\end{aligned}
$$

Substituting (23) into our dynamics (19) and using (24), we obtain a two point boundary value problem:

$$
\begin{array}{r}
\left\{\begin{array}{c}
\dot{x} \\
\dot{\lambda}
\end{array}\right\}=\left[\begin{array}{cc}
A & \frac{1}{\gamma} \hat{F}_{1} M_{2} \hat{F}_{1}^{T} \\
-C^{T}\left(\hat{H}_{1}^{T} Q_{1} \hat{H}_{1}^{T}-\gamma N_{1}^{T} R_{1} N_{1}-\gamma V^{-1}\right) C & -A^{T}
\end{array}\right]\left\{\begin{array}{c}
x \\
\lambda
\end{array}\right\}+ \\
\left\{\begin{array}{cc}
0 \\
C^{T}\left(\hat{H}_{1}^{T} Q_{1} \hat{H}_{1}^{T}-\gamma N_{1}^{T} R_{1} N_{1}\right) C \hat{x}-\gamma C^{T} V^{-1} y
\end{array}\right\} .
\end{array}
$$

If we assume solutions $x^{*}$ and $\lambda^{*}$ to (27) and a quadratic form of the optimal return function, then:

$$
\lambda^{*}=\Pi\left(x^{*}-x_{p}\right)
$$

where $x_{p}$ is a measurement dependent variable which will be shown to reduce to the estimate of the optimal state. Using (28) and the first equation of (27), the second equation of (27) becomes:

$$
\begin{array}{r}
0=\left[\dot{\Pi}+A^{T} \Pi+\Pi A+\frac{1}{\gamma} \Pi \dot{F}_{1} M_{2} \dot{F}_{1}^{T} \Pi+C^{T}\left(\hat{H}_{1}^{T} Q_{1} \hat{H}_{1}^{T}-\gamma N_{1}^{T} R_{1} N_{1}-\gamma V^{-1}\right) C\right] x^{*} \\
-\dot{\Pi} x_{p}-\Pi x_{p}-A^{T} \Pi x_{p}-C^{T}\left(\hat{H}_{1}^{T} Q_{1} \hat{H}_{1}^{T}-\gamma N_{1}^{T} R_{1} N_{1}\right) C \hat{x}+\gamma C^{T} V^{-1} y
\end{array}
$$

Now, add and subtract. $\gamma C^{T} V^{-1} C \hat{x}$ and $\left[\Pi A+C^{T}\left(\hat{H}_{1}^{T} Q_{1} \hat{H}_{1}^{T}-\gamma N_{1}^{T} R_{1} N_{1}-\gamma V^{-1}\right) C\right] x_{p}$ to (29):

$$
\begin{aligned}
& 0=\left[\dot{\Pi}+A^{T} \Pi+\Pi A+\frac{1}{\gamma} \Pi \hat{F}_{1} M_{2} \hat{F}_{1}^{T} \Pi+C^{T}\left(\hat{H}_{1}^{T} Q_{1} \hat{H}_{1}^{T}-\gamma N_{1}^{T} R_{1} N_{1}-\gamma V^{-1}\right) C\right]\left(x^{*}-x_{p}\right) \\
& -\Pi \dot{x}_{p}-\Pi A x_{p}-\left[C^{T}\left(\hat{H}_{1}^{T} Q_{1} \hat{H}_{1}^{T}-\gamma \lambda_{1}^{T} R_{1} N_{1}-\gamma C^{T} V^{-1}\right) C\right]\left(\dot{x}-x_{p}\right)+\gamma C^{T} V^{-1}(y-C \hat{x}) .
\end{aligned}
$$

Thus, if we set:

$$
\begin{aligned}
-\dot{\Pi} & =A^{T} \Pi+\Pi A+\frac{1}{\gamma} \Pi \dot{F}_{1} M_{2} \dot{F}_{1}^{T} \Pi+C^{T}\left(\dot{H}_{1}^{T} Q_{1} \hat{H}_{1}^{T}-\gamma N_{1}^{T} R_{1} N_{1}-\gamma V^{-1}\right) C \\
\Pi \dot{x}_{n} & =\Pi A x_{p}-C^{T}\left(\dot{H}_{1}^{T} Q_{1} \dot{H}_{1}^{T}-\gamma N_{1}^{T} R_{1} N_{1}-\gamma V^{-1}\right) C\left(\dot{x}-x_{p}\right)+\gamma C^{T} V^{-1}(y-C \hat{x}) .
\end{aligned}
$$

(30) is satisfied identically. (31) is all estimator Riccati Equation. If we sett: 


$$
\Pi=\gamma P^{-1}
$$

we can convert. (31) into:

$$
\dot{P}=P A^{T}+P A-P C^{T}\left(V^{-1}+N_{1}^{T} R_{1} N_{1}-\frac{1}{\gamma} \hat{H}_{1}^{T} Q_{1} \hat{H}_{1}^{T}\right) C P+\hat{F}_{1} M_{2} \dot{F}_{1}^{T}
$$

which is a Riccati Equation of the sort seen in [12],[13], and [14]. (32) looks like an estimator, but its final form will not become apparent until we solve the second half of the game problem.

\subsection{Minimization with Respect to $\dot{x}$ and Maximization with Respect to $v$}

The first part of our game solution led to optimal values for $\mu$ and $x\left(t_{0}\right)$ :

$$
\begin{aligned}
u^{*} & =\frac{1}{\gamma} \hat{F}_{1} M_{2} \hat{F}_{1}^{T} \lambda \\
x\left(t_{0}\right)^{*} & =\Pi_{0}^{-1} \lambda\left(t_{0}\right)+\hat{x}_{0}
\end{aligned}
$$

If we substitute these optimal values into the cost function (17) we obtain a new cost, $\bar{J}$, which is written as:

$$
\bar{J}=\int_{t_{0}}^{t_{1}}\left[\|(x-\hat{x})\|_{C^{T}\left(\dot{H}_{1}^{T} Q_{1} \dot{H}_{1}-\gamma N_{1}^{T} R_{1} N_{1}\right) C}^{2}-\|\lambda\|_{\frac{1}{\gamma} \dot{F}_{2} \mathcal{H}_{2} \dot{F}_{1}^{T}}^{2}-\gamma\|y-C x\|_{V-1}^{2}\right] d t-\left\|\lambda\left(t_{0}\right)\right\|_{\Pi_{0}^{-1}}^{2}
$$

Maximization of the cost with respect to $v$ is unnecessary at this point. Since $v=y-C x$ and $y$ is a given measurement vector, $v^{*}$ is determined once $x^{*}$ has been. Hence, the second part of the solution reduces to a single minimization:

$$
\min _{\dot{\xi}} \bar{J} \leq 0
$$

To begin, we add the identically zero term:

$$
\left\|\lambda\left(t_{1}\right)\right\|_{\Pi\left(t_{1}\right)^{-1}}^{2}-\left\|\lambda\left(t_{1}\right)\right\|_{\Pi\left(t_{1}\right)^{-1}}^{2}+\int_{t_{1}}^{t_{1}} \frac{d}{d t}\|\lambda(t)\|_{\Pi^{-1}}^{2} d t=0
$$

to (37). After applying the houndary condition for $\lambda$ at. $t_{1}(25)$ and carrying out the differentiation of the $\|\lambda\|_{n-1}^{2}$ term, we get:

$$
\begin{aligned}
\bar{J}=\int_{t_{11}}^{t_{1}}\left[\|(x-\tilde{x})\|_{C^{T}\left(\dot{H}_{1}^{T} Q_{1} \dot{H}_{1}-\gamma N_{1}^{T} R_{1} N_{1}\right) C}^{2}-\|\lambda\|_{\frac{1}{\gamma} \dot{F}_{1} \Lambda I_{2} \dot{F}_{1}^{T}-\gamma\|y-C x\|_{V^{-1}}^{2}}^{2}\right. \\
\left.+\dot{\lambda}^{T} \Pi^{-1} \lambda^{T}+\lambda^{T} \dot{\Pi}^{-1} \lambda+\lambda^{T} \Pi^{-1} \dot{\lambda}\right] d t+\left\|\lambda\left(t_{1}\right)\right\|_{\Pi^{-1}\left(t_{11}\right)-\Pi_{0}^{-1}}^{2}
\end{aligned}
$$

Note that (38) gives us a houmdary coudition for $(31)$ : 


$$
\Pi\left(t_{0}\right)=\Pi_{0}
$$

Applying this boundary condition and sulstituting the differential equation for $\lambda,(24)$, into (38) leads to:

$$
\begin{array}{r}
\bar{J}=\int_{t_{0}}^{t_{1}}\left[\lambda^{T}\left(-A \Pi^{-1}-\Pi^{-1} A^{T}-\hat{F}_{1} M_{2} \hat{F}_{1}^{T}+\dot{\Pi}^{-1}\right) \lambda+(x-\hat{x})^{T} C^{T}\left(\hat{H}_{1}^{T} Q_{1} \hat{H}_{1}-\gamma N_{1}^{T} R_{1} N_{1}\right) C(x-\hat{x})\right. \\
-(x-\hat{x})^{T} C^{T}\left(\hat{H}_{1}^{T} Q_{1} \hat{H}_{1}-\gamma N_{1}^{T} R_{1} N_{1}\right) C \Pi^{-1} \lambda-\lambda^{T} \Pi^{-1} C^{T}\left(\dot{H}_{1}^{T} Q_{1} \hat{H}_{1}-\gamma N_{1}^{T} R_{1} N_{1}\right) C(x-\hat{x})- \\
\gamma\left[(y-C x)^{T} V^{-1}(y-C x)+(y-C x)^{T} V^{-1} C \Pi^{-1} \lambda+\lambda^{T} \Pi^{-1} C^{T} V^{-1}(y-C x)\right] d t .
\end{array}
$$

From (31) the differentiai equation for $\Pi^{-1}$ is:

$$
\dot{\Pi}^{-1}=-\Pi^{-1} \dot{\Pi} \Pi^{-1}=\Pi^{-1} A^{T}+A \Pi^{-1}+\frac{1}{\gamma} \hat{F}_{1} M_{2} \hat{F}_{1}^{T}+\Pi^{-1} C^{T}\left(\hat{H}_{1}^{T} Q_{1} \hat{H}_{1}-\gamma N_{1}^{T} R_{1} N_{1}-\gamma V^{-1}\right) C \Pi^{-1}
$$

After we insert (41) into (40) and cancel terms, we are left with what turns out to be a pair of quadratic terms:

$$
\begin{array}{r}
\bar{J}=\int_{t_{0}}^{t_{1}}\left\{\left[\Pi^{-1} \lambda-(x-\hat{x})\right]^{T} C^{T}\left(\hat{H}_{1}^{T} Q_{1} \hat{H}_{1}-\gamma N_{1}^{T} R_{1} N_{1}\right) C\left[\Pi^{-1} \lambda-(x-\hat{x})\right]-\right. \\
\left.\gamma\left[C \Pi^{-1} \lambda+(y-C x)\right]^{T} V^{-1}\left[C \Pi^{-1} \lambda+(y-C x)\right]\right\} d t
\end{array}
$$

Now, use the solution for the optimal value of $\lambda(28)$ and substitute into (42) to get:

$$
\bar{J}=\int_{t_{1}}^{t_{1}}\left[\left(\dot{x}-x_{p}\right)^{T} C^{T}\left(\hat{H}_{1}^{T} Q_{1} \dot{H}_{1}-\gamma N_{1}^{T} R_{1} N_{1}\right) C\left(\dot{x}-x_{p}\right)-\gamma\left(y-C x_{p}\right)^{T} V^{-1}\left(y-C x_{p}\right)\right] d t .
$$

Because of the projectors $\dot{H}_{1}$ and $N_{1}$, the minimizing value of $\dot{x}$ is:

$$
\tilde{x}^{*}=x_{p} \bmod \operatorname{ker} \hat{H}_{1} C
$$

Using (44) as a guide, we rewrite (32) as:

$$
\Pi \dot{x}=\Pi A \hat{x}^{*}+\gamma C^{T} V^{-1}\left(y-C \hat{x}^{*}\right)
$$

In (45) we have used $\dot{j}^{-}=x_{p}$ instead of (44). However, this choice is justified in that an adjustment of (32) in the manner suggested hy (44) would huild in a bias to our estimate which would be better in the sense of the game but not. in our ultimate oljective which is to design a fault detection filter. Since $\Pi$ is positive-definite for $\gamma>0$, we can rewrite (45) as:

$$
\dot{x}^{-}=A \dot{x}^{*}+\gamma \Pi^{-1} C^{T} V^{-1}\left(y-C \hat{x}^{*}\right)
$$


Alternatively, the analyst conkl use (34) and:

$$
\ddot{\dot{x}}=A \dot{s}^{*}+P C^{T} V^{-1}\left(y-C \dot{x}^{*}\right) \text {. }
$$

This form of the filter is equivalent. 10 (46). however; experience has shown that numerical problems are more likely to be seen when trying to find a solution to (34) than (31) when $\gamma$ is brought to extremely small values. For convenience, we will use $\dot{x}$ instead of $\dot{x}^{-}$when referring to the optimal state estimate with the understanding that it is the estimate that comes from the game solution which is being used.

From (44) we can see how this game filter mimics a fault detection filter. $\hat{x}$ does better by inducing estimation errors in the space defiaed by the projector $\ddot{H}_{1}$ than by trying to estimate the state exactly. This implies that knowledge of the time history of $i_{2}$, either by measurement or estimation, is not needed.

The solution of the fault. detection filter problem exists at the limit of the game solution when $\gamma$ is taken to zero. Finding the solution or even showing that it exists is not a straightforward matter. In both versions of the game Riccati Equation (31) and (34), there are terms which go to infinity as $\gamma$ goes to zero. Kwakernaak and Sivan [15], studied a similar situation for the linear cuadratic regulator. The linear-quadratic cost function in their problem, however, is always non-negative while our game cost can be both positive and negative. Hence, their results are not directly applicable here. Also, it is well known [14] that for game Riccati Equations, there is a minimum value of $\gamma$ for which the equation will yield a positive-definite solution. Below this critical value of $\gamma$ any number of different phenomena could occur (e.g. eigenvalues on the imaginary axis) which make positive-definite solutions impossible.

It. t.urns out. that we can resolve the second difficulty fairly quickly. By decreasing the noise weighting $V$ to zero along with $\gamma$-i.e. $V-0$ as $\gamma \rightarrow 0$ - we can fund solutions to $(31)$ and $(34)$ for smaller and smaller $\gamma$. What we get in the limit turns out to be a siugular filteriug problem. This insight, in fact, is the key to resolving the first difficulty. By using a pair of techuiques from singular optimal control theory in the next. section, we will be able to show the conditions for the existence of the limiting solution and the nature of this solution when it exists.

\section{The Limiting Case Solution via Singular Optimal Control Techniques}

\subsection{Conditions for the Nou-Positivity of the Gane Cost: An LMI for the Gaue}

In this section, we will find snfficient conditions for the non-positivity of the game cost. These conditions fall out when we manipulate the cost slightly and then set. $\dot{s}$ to its optimal strategy found in Section 2 . The game cost. then becomes a single quadratic form: 


$$
J\left(\dot{x}, x\left(t_{11}\right), i_{2}, v\right)=\int_{t_{10}}^{t_{1}} \xi^{T} \bar{W} \xi d t
$$

where $\xi$ is some vector consisting of linear combinalions of the game players. The non-negativity of the cost then hinges on the sign definiteness of $\bar{W}$, giving rise to a linear matrix inequality. Historically, this technique was first seen in the singular optimal control theory [16] and [17] and the derivation seen here follows in that vein

We begin with the cost. function as given by $(20)$. Note that the $(x-\hat{x})$ terms have been combined:

$$
J=\int_{t_{0}}^{t_{1}}\left[\|(x-\dot{x})\|_{C^{T}\left(\hat{H}_{1}^{T} Q_{1} \dot{H}_{1}-\gamma N_{1}^{T} R_{1} N_{1}\right) C}^{2}-\gamma\left\|\dot{\mu}_{2}\right\|_{M_{2}^{-1}}^{2}-\gamma\|y-C x\|_{V-1}^{2}\right] d t-\left\|x\left(t_{0}\right)-\dot{x}_{0}\right\|_{\Pi_{0}}^{2} .
$$

We now append the dynamics of the system to (49) through the Lagrange Multiplier $(x-\hat{x})^{T} \Pi$ :

$$
\begin{array}{r}
J=\int_{t_{0}}^{t_{1}}\left[\|(x-\bar{x})\|_{C^{T}\left(\dot{H}_{1}^{T} Q_{1} \dot{H}_{1}-\gamma N_{1}^{T} R_{1} N_{1}\right) C}^{2}-\gamma\left\|\hat{\mu}_{2}\right\|_{M_{2}^{-1}}^{2}-\gamma\|y-C x\|_{V-1}^{2}+\right. \\
\left.(x-\hat{x})^{T} \Pi\left(A x+\hat{F}_{1} \bar{\mu}_{2}-\dot{x}\right)\right] d t-\left\|x\left(t_{0}\right)-\hat{x}_{0}\right\|_{\Pi_{0}}^{2} .
\end{array}
$$

Add and subtract to $(21)$ the terms $(x-\hat{x})^{T} \Pi A \hat{x}$ and $(x-\hat{x})^{T} \Pi \dot{\hat{x}}$. Collect terms to get:

$$
\begin{gathered}
J=\int_{t_{0}}^{t_{1}}\left\{\|(x-\hat{x})\|_{\Pi A+C^{T}\left(\dot{H}_{1}^{T} Q_{1} \dot{H}_{1}-\tau N_{1}^{T} R_{1} N_{1}\right) C}^{2}-\gamma\left\|\hat{\mu}_{2}\right\|_{M_{2}^{-1}}^{2}-\gamma\|y-C x\|_{V-1}^{2}\right. \\
\left.+(x-\hat{x})^{T} \Pi \hat{F}_{1} \dot{\mu}_{2}-(x-\hat{x})^{T} \Pi(\dot{x}-\dot{\hat{x}})+(x-\hat{x})^{T}[\Pi A \hat{x}-\Pi \dot{\hat{x}}]\right\} d t-\left\|x\left(t_{0}\right)-\dot{x}_{0}\right\|_{\Pi_{0}}^{2}
\end{gathered}
$$

Note, we have moved $\Pi A$ into the weighting $\|(x-\hat{x})\|^{2}$. As we continue along, new terms will be appear in the weighting of $\|(x-\hat{x})\|^{2}$ as we manipulate the cost function. Integrate $(x-\dot{j})^{T} \Pi(\dot{x}-\dot{\hat{x}})$ by parts:

$$
\begin{aligned}
& J=\int_{t_{0}}^{t_{1}}\left\{\|(x-\dot{x})\|_{\tilde{n}+\Pi A+C^{T}\left(\dot{H}_{1}^{T} Q_{1} \dot{H}_{1}-\gamma N_{1}^{T} R_{1} N_{1}\right) C}^{2}-\gamma\left\|\dot{i}_{2}\right\|_{M_{2}-1}^{2}-\gamma\|y-C x\|_{\nu-1}^{2}+(x-\dot{x})^{T} \Pi \hat{F}_{1} \hat{H}_{2}\right. \\
& \left.+(x-\hat{x})^{T}[\Pi . A \dot{x}-\Pi \dot{x}]+(\dot{x}-\dot{x})^{T} \Pi(x-\dot{x})\right\} d t-\left\|x\left(t_{0}\right)-\dot{x}_{0}\right\|_{\Pi_{11}}^{2}-\Pi\left(t_{11}\right)-\left\|x\left(t_{1}\right)-\hat{x}\left(t_{1}\right)\right\|_{\Pi\left(t_{1}\right)}^{2}
\end{aligned}
$$

Substitute the state equation for $\dot{x}(19)$ and add and subtract $\hat{x}^{T} A^{T} \Pi(x-\dot{j})$ :

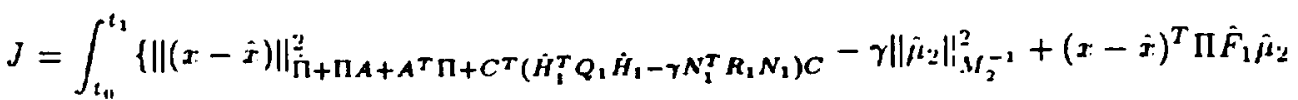

$$
\begin{aligned}
& +\mu_{1}^{T} \dot{F}_{1}^{T} \Pi(x-\hat{x})^{T}-\gamma\|y-C x\|_{V-1}^{2}+(x-\hat{x})^{T}[-\Pi \dot{\dot{x}}+\Pi A \dot{x}]+[-\Pi \dot{\hat{x}} \\
& \left.+\Pi A \dot{x}]^{T}(s-\hat{x})\right\} d t-\left\|x\left(t_{0}\right)-\dot{x}_{0}\right\|_{\Pi_{1}-\Pi\left(t_{0}\right)}^{2}-\left\|x\left(t_{1}\right)-x\left(\hat{t}_{1}\right)\right\|_{\Pi\left(t_{1}\right)}^{2} .
\end{aligned}
$$

We are now going to manipulate the $\|y-C x\|_{V-1}^{2}$ term by adding and subtracting $C$ s inside of the term so that it reads $\|(y-C \hat{x})-C(x-\hat{x})\|_{\hat{v}-1}^{j}$. Expand this duadratic term out and collect terms so that we end up with: 


$$
\begin{aligned}
& J=\int_{t_{\mathrm{n}}}^{z_{1}}\left\{\|(x-\hat{x})\|_{\dot{\mathrm{n}}+\Pi A+A^{T} \Pi+C^{T}\left(\dot{H}_{1}^{T} Q_{1} \dot{H}_{1}-\gamma N_{1}^{T} R_{1} N_{1}-\gamma V-1\right) C}^{2}-\gamma\left\|\hat{\mu}_{2}\right\|_{M_{2}^{-1}}^{2}+(x-\hat{x})^{T} \Pi \hat{F}_{1} \hat{\mu}_{2}\right. \\
& +\hat{\mu}_{1}^{T} \dot{F}_{1}^{T} \Pi(x-\hat{x})^{T}-\gamma\|\underline{y}-C \hat{x}\|_{V-1}^{2}+(x-\hat{x})^{T}\left[-\Pi \dot{\hat{x}}+\Pi A \hat{x}+\gamma C^{T} V^{-1}(y-C \dot{x})\right] \\
& \left.-\left[\Pi \dot{\bar{x}}+\Pi A \hat{x}+\gamma C^{T} V^{-1}(y-C \dot{x})\right]^{T}(x-\hat{x})\right\} d t-\left\|x\left(t_{0}\right)-\hat{x}_{0}\right\|_{\Pi_{0}-\Pi\left(t_{0}\right)}^{2}-\left\|x\left(t_{1}\right)-\hat{x}\left(t_{1}\right)\right\|_{\Pi\left(t_{2}\right)}^{2}
\end{aligned}
$$

Using (45) we can eliminate a pair of terms in (54). We are then left with a quadratric in the form:

$$
J=\int_{t_{\omega}}^{t_{1}} \xi^{T} \bar{W} \xi d t-\left\|x\left(t_{0}\right)-\hat{x}_{0}\right\|_{\Pi_{u}-\Pi\left(t_{0}\right)}^{2}-\left\|x\left(t_{1}\right)-x\left(\hat{t}_{1}\right)\right\|_{\Pi\left(t_{1}\right)}^{2},
$$

where:

$$
\xi=\left\{\begin{array}{c}
\left(x-\tilde{x}_{1}\right) \\
\hat{\mu}_{2} \\
(y-C \dot{x})
\end{array}\right\}
$$

and:

$$
\bar{W}:=\left[\begin{array}{ccc}
C^{T}\left(\hat{H}_{1}^{T} Q_{1} \hat{H}_{1}-\gamma V^{-1}-\gamma N_{1}^{T} R_{1} N_{1}\right) C+A^{T} \Pi+\Pi A+\dot{\Pi} & \Pi \hat{F}_{1} & 0 \\
\hat{F}_{1}^{T} \Pi & -\gamma M_{2}^{-1} & 0 \\
0 & 0 & -\gamma V^{-1}
\end{array}\right] .
$$

Define the upper $2 \times 2$ block of $\bar{W}$ as:

$$
W(\Pi):=\left[\begin{array}{cc}
C^{T}\left(\hat{H}_{1}^{T} Q_{1} \hat{H}_{1}-\gamma V^{-1}-\gamma N_{1}^{T} R_{1} N_{1}\right) C+A^{T} \Pi+\Pi A+\dot{\mathrm{M}} & \Pi \hat{F}_{1} \\
\hat{F}_{1}^{T} \Pi & -\gamma M_{2}^{-1}
\end{array}\right]
$$

For matrices $\Pi \geq 0$ such that:

$$
\begin{aligned}
W(\Pi) & \leq 0 \\
\Pi_{0}-\Pi\left(t_{1}\right) & \geq 0 \\
\Pi\left(t_{1}\right) \geq 0 &
\end{aligned}
$$

$\bar{W}$ is clearly negative semi-definite. Hence, we need only pay attention to the smaller LMI, $W(\mathrm{I})$.

For $\gamma>0$, it is easy to see that the Riccati Equation (31) of the previous section is embedded in (56). In fact, the solution of $(31)$ is the solution of $W(\Pi)$ which minimizes its rank [18]. Thus with (50) and (45), we retain the results of the previous section, but. in a form which can be easily analyzed in the limit $\gamma \rightarrow 0$. If we define $\bar{V}=\lim _{\urcorner},:$: sufficient conditions for $J \leq 0$ in the limit as $\gamma \rightarrow 0$ are:

$$
\begin{aligned}
\Pi \dot{F}_{1} & =0 \\
\dot{\Pi}+A^{T} \Pi+\Pi A+C^{T}\left(\hat{H}_{1}^{T} Q_{1} \hat{H}_{1}-\bar{V}^{-1}\right) C & \leq 0 .
\end{aligned}
$$


along with the houmdary conditions (58) and (59).

(60) clearly shows that in the limit, the Riccati Mat,rix, II, ohtains a non-t.rivial mull space which contains the image of the nuisance failure map, $\dot{F}_{1}$. Moreover, those familiar with singular optimal control theory will recognize (60) and (61) as conditions seen previously for the singular LQ regulator (see e.g. [16]). This tells us, first of all, that the limiting form of this game filter is a singular filter. It is likely that similar results hold for game theoretic $\left(H_{\infty}\right)$ filters and controllers in general. Secondly, singular optimal control provides a wealth of results and insights which we can apply to the analysis of this filter. This is, in fact, what we will do next.

\subsection{A Riccati Equation for the Limiting Form of the Game Theoretic Filter}

Goh (see [11]) used a transformation on the control input space to derive a full-order Riccati Equation for the totally singular linear quadratic regulator problem. By applying the same type of transformation on the nuisance fault input space, we can obtain a similar equation for the limiting game filter. The existence of the solution to this equation gives the condition for the existence of the game solution in the limit. We have already seen this transformation applied to the construction of the time-varyiug projector, $\hat{H}_{1},(11)$. Because this Riccati Matrix must also have a non-trivial null space, we will not be able to use the solution to this Riccati Equation directly in a game filter, but. this matrix will also prove to be useful when we look at reduced-order filters.

We start with the game cost for the limiting case:

$$
J^{*}=\lim _{\gamma \rightarrow 0} J=\int_{t_{0}}^{t_{1}}\|x-\hat{x}\|_{C^{\top} \dot{H}_{1}^{T} Q_{1} \hat{H}_{1} C}^{2}-\|y-C x\|_{\bar{V}^{-1}}^{2} d t,
$$

where $\bar{V}^{-1}:=\lim _{\gamma \rightarrow 0}(\gamma V)^{-1}$. Now, define a new muisance fault vector, $\rho_{1}$ and a new st.ate vector, $\alpha_{1}$ :

$$
\begin{aligned}
& \rho_{1}:=\int_{t_{0}}^{t} \hat{\mu}_{2} d t \\
& \alpha_{1}:=x-\hat{F}_{1} \rho_{1} \equiv x-B_{1} \rho_{1}
\end{aligned}
$$

Note that we have defined a matrix $B_{1}:=\hat{F}_{1}$. The reason for the numbered subscripts will become apparent later. Differentiating (6;3), we get:

$$
i_{1}=A a_{1}+\left(A B_{1}-\dot{B}_{1}\right) \mu_{1}
$$

as a new state equation and: 


$$
\begin{aligned}
J^{*}= & \int_{t_{11}}^{t_{1}}\left[\left\|\alpha_{1}-\dot{x}\right\|_{C^{T} \eta_{1}^{T} Q_{1} H_{2} C}^{2}+\left(\alpha_{1}-\dot{z}\right)^{T} C^{T} \hat{H}_{1}^{T} Q_{1} \hat{H}_{1} C B_{1} \rho_{1}+\rho_{1}^{T} B_{1}^{T} C^{T} \hat{H}_{1}^{T} Q_{1} \hat{H}_{1} C B_{1} \rho_{1}-\right. \\
& \left\|y-C \alpha_{1}\right\|_{V^{-1}}^{2}-\left(y-C \alpha_{1}\right)^{T} \bar{V}^{-1} C B_{1} \rho_{1}-\rho_{1}^{T} B_{1}^{T} C^{T} \bar{V}^{-1}\left(y-C\left(\alpha_{1}\right)-\left\|\rho_{1}\right\|_{B_{1}^{T} C^{T} \bar{V}^{-1} C B_{1}}^{2}\right] d t
\end{aligned}
$$

as the new game cost. Because $\hat{H}_{1}$ is a projector constructed so that. $\hat{H}_{1} C \bar{F}_{1}=0$, we can simplify (65) to:

$$
\begin{aligned}
& J^{*}=\int_{t_{11}}^{t_{1}}\left[\left\|\alpha_{1}-\hat{x}\right\|_{C^{T} \dot{H}_{1}^{T} Q_{1} \hat{H}_{2} c}^{2}-\left\|y-C \alpha_{1}\right\|_{\bar{V}-1}^{2}-\left(y-C \alpha_{1}\right)^{T} \bar{V}^{-1} C B_{1} \rho_{1}\right. \\
& \left.-\rho_{1}^{T} B_{1}^{T} C^{T} \bar{V}^{-1}\left(y-C \alpha_{1}\right)-\left\|\rho_{1}\right\|_{B_{1}^{T} C^{T} \bar{v}^{-1} C B_{1}}^{2}\right] d t .
\end{aligned}
$$

Now, if $B_{1}^{T} C^{T} \bar{V}^{-1} C B_{1}>0$, we can solve the following differential game:

$$
\min _{\dot{i}} \max _{\rho_{i}} J^{*} \leq 0
$$

subject to (64). Because of its similarity to the derivation given in section 3, we do not provide the solution here. But if the interested reader follows the steps in section $3^{2}$, he will find that the solution leads to a Riccati equation:

$$
\begin{array}{r}
-\dot{S}=S A+A^{T} S+C^{T}\left(\hat{H}_{1}^{T} Q_{1} \hat{H}_{1}-\bar{V}^{-1}\right) C+\left[S\left(A B_{1}-\dot{B}_{1}\right)-C^{T} \bar{V}^{-1} C B_{1}\right] \\
\times\left(B_{1}^{T} C^{T} \bar{V}^{-1} C B_{1}\right)^{-1}\left[\left(A B_{1}-\dot{B}_{1}\right)^{T} S-B_{1}^{T} C^{T} \bar{V}^{-1} C\right]
\end{array}
$$

with the boundary condition:

$$
S\left(t_{0}\right)=0
$$

It may happen, however, that $C B_{1}=0$, which would make $B_{1}^{T} C^{T} \bar{V}^{-1} C B_{1}=0$ and which would invalidate our Riccati Equation (67). The remedy to this situation is to perform the same transformation as before but on the $\rho_{1}$ input space via the recursion equations:

$$
\begin{aligned}
& \mu_{i}=\int_{t_{11}}^{t} \rho_{i-j} d t \\
& B_{i}=A B_{i-1}-\dot{B}_{i-1} \\
& a_{i}=x-B_{i} \rho_{i} .
\end{aligned}
$$

The process stopls once a $B_{i}$ is found such that $C B_{i} \neq 0$. The game is then:

${ }^{2}$ Hint: the first step involves converting $y-C a$ intu $(y-C \dot{x})+C(\alpha-\dot{x})$ 


$$
\begin{array}{r}
\min _{\dot{i}} \max _{\rho_{i}} J^{*}=\int_{t_{\omega}}^{t_{1}}\left[\left\|\boldsymbol{r}_{i}-\bar{x}\right\|_{C^{T} \dot{H}_{1}^{T} Q_{1} H_{1} C}^{2}-\| y-C\left(\alpha_{i} \|_{\bar{V}^{-1}}^{2}-\left(y-C a_{i}\right)^{T} \bar{V}^{-1} C B_{i} \rho_{i}\right.\right. \\
\left.-\rho_{i}^{T} B_{i}^{T} C^{T} \bar{V}^{-1}\left(y-C \alpha_{i}\right)-\left\|\rho_{i}\right\|_{B_{i}^{T} C^{T} \bar{V}^{-1} C B_{i}}^{2}\right] d t
\end{array}
$$

sulject to:

$$
\dot{\alpha}_{i}=A \alpha_{i}+\left(A B_{i}-\dot{B}_{i}\right) \rho_{i}
$$

The general form of the Goh Riccati Equation is then:

$$
\begin{array}{r}
-\dot{S}=S A+A^{T} S+C^{T}\left(\dot{H}_{1}^{T} Q_{1} \dot{H}_{1}-\bar{V}^{-1}\right) C+\left[S\left(A B_{i}-\dot{B}_{i}\right)-C^{T} \bar{V}^{-1} C B_{i}\right] \\
\times\left(B_{i}^{T} C^{T} \bar{V}^{-1} C B_{i}\right)^{-1}\left[\left(A B_{i}-\dot{B}_{i}\right)^{T} S-B_{i}^{T} C^{T} \bar{V}^{-1} C\right]
\end{array}
$$

The following theorem shows that (74) is a Riccati Equation for the limiting form of the game theoretic filter.

Theorem 4.1. The solution $S$ to (74) satisfies the sufficient conditions for the non-positivity of the game cost: Equations 60 and 61.

Proof. (The proof given here follows Bell and Jacobson [16] (pg. 121). Due to its importance, we list it here.) Clearly, (74) implies that:

$$
\dot{S}+S A+A^{T} S+C^{T}\left(\hat{H}_{1}^{T} Q_{1} \dot{H}_{1}-\bar{V}^{-1}\right) C \leq 0, \forall t \in\left[t_{1}, t_{1}\right]
$$

which is (60). Now, pre-multiply (74) by $B_{i}$ and add $-\dot{B}_{i}^{T} S$ to both sides of the resulting equation to get:

$$
\begin{aligned}
-B_{i}^{T} \dot{S}-\dot{B}_{i}^{T} S=B_{i}^{T} S A-\dot{B}_{i} S+B_{i}^{T} A^{T} S- & B_{i}^{T} C^{T} \bar{V}^{-1} C+\left[B_{i}^{T} S\left(A B_{i}-\dot{B}_{i}\right)-B_{i}^{T} C^{T} \bar{V}^{-1} C B_{i}\right] \\
& \times\left(B_{i}^{T} C^{T} \bar{V}^{-1} C B_{i}\right)^{-1}\left[\left(A B_{i}-\dot{B}_{i}\right)^{T} S-C^{T} \bar{V}^{-1} C B_{i}\right]
\end{aligned}
$$

Rearranging terms leads to a differential equation in $B_{i}^{T} S$ with $(68)$ as the boundary condition:

$$
-\frac{d}{d t}\left[B_{i}^{T} S\right]=B_{i}^{T} S\left[A+B_{i}^{T} S\left(A B_{i}-\dot{B}_{i}\right)\left(B_{i}^{T} C^{T} \bar{V}^{-1} C B_{i}\right)^{-1}\left[\left(A B_{i}-\dot{B}_{i}\right)^{T} S-C^{T} \bar{V}^{-1} C B_{i}\right] .\right.
$$

The solution to (77) given $(68)$ is:

$$
B_{i}^{T}(t) S(t)=0, \forall t \in\left[t_{1}, t_{1}\right]
$$

The necessary condition (60) actually requires that $\hat{F}_{1}^{T} S(t)=0$. However, since $B_{1}=\hat{F}_{1}$ and with the following proposition, (60) is satisfied. 
Proposition 4.2. Let $i \in \mathbb{N}$ be the smallest number such that. $C B_{i} \neq 0$. Then, the solution, $S$, to (110) is such that

$$
S B_{j}=0, \forall j \leq i, \forall t \in\left[t_{0}, t_{1}\right]
$$

Proof. That $S B_{i}=0$ is a result of the solution to the game and is analogous to the $S \hat{F}_{1}=0$ condition found in the solution to the original game. The proof that $S B_{j}=0$ for all $j<i$ can be found in [19]. The proof used there is an inductive argument which applies the same steps used above in Theorem 6.1 to prove $S B_{i}=0 \forall t \in\left[t_{0}, t_{1}\right]$ to show that the same holds for all $j \leq i$.

\section{The Equivalence of the Game Theoretic Filter to the Beard-Jones Filter in the Limit}

In this section, we relurn to time-invariant case and slow that for these systems the solution to the fault det.ection filter problem as stated in Definition 2.2 also solves the problem as stated by Definition 2.1 - i.c. limiting form of the game theoretic filter is a Beard-Jones Fault Detection Filter. The required invariant subspace is the kernal of II. To prove to this assertion, we first need a minor lemma:

\section{Lemma 5.1. (ker $\Pi \cap \operatorname{ker} C) \subset \operatorname{ker} \dot{\Pi}$}

Proof. Let. $x \in$ ker $\Pi \cap$ ker $C$. Pre-multiply $(61)$ by $x^{T}$ and post-multiply by $x$. This leaves:

$$
x^{T} \dot{n} x \leq 0
$$

Since $\dot{\text { II }}$ can be negative or positive-definite, this can be true in general if and only if:

$$
x^{T} \dot{\Pi} x=0
$$

which implies onr proposition.

Theorem 5.2. kerl is a sulispace which solves the fault detection filter problem

Proof. The three conditions listed by Definition 2.1 are subspace inclusion (Equation 4), ont.put. separability (Equation 5), and $(C, A)$-iuvariance (Equation $(6)$. (60) clearly implies subspace iuclusion. Since we are trying to detect only one fault, output separahility is satisfied trivially. Thus, all that remains is to show (C:A)-invariance. 
From Wonlıam [10], a necessary and sufficient condition for kerll to be $(C, A)$-invariant is that:

$$
A(\operatorname{ker} \Pi \cap \operatorname{ker} C) \subset \operatorname{ker} \Pi
$$

Therefore, let $x \in A(\operatorname{ker} \Pi \cap \operatorname{ker} C)$. That is, there exists a vector $\varsigma$ such that:

$$
x=A \varsigma \text { and } \Pi \varsigma=C \varsigma=0 .
$$

Now consider (61). If we post-multiply (61) by $\varsigma$ and use our lemma, we get:

$$
\Pi A \varsigma \leq 0 \Rightarrow \varsigma^{T} A^{T} \Pi A \varsigma \leq 0 .
$$

Since $\Pi \geq 0$, this means that:

$$
\varsigma^{T} A^{T} \Pi A \varsigma=0
$$

which implies that:

$$
\Pi A \varsigma=\Pi x=0 \Rightarrow x \in \operatorname{ker} \Pi
$$

From this we conclude that $A(\operatorname{ker} \Pi \cap \operatorname{ker} C) \subset \operatorname{ker} \Pi$ and so $\operatorname{ker} \Pi$ is $(C, A)$-invariant.

Remark 1. In actual practice, it is not necessary to use the limiting form of the filter. In many $H_{\infty}$ designs, $\gamma$ is not taken to its smallest possible value, but left at one which results in an acceptable compromise between all of the (usually competing) design ohjectives. The virtue of a game theoretic approach to fault detection filter design is that it gives the designer a "knob" with which he can make the filter more like a Beard-Jones filter (small $\gamma$ and small $V$ ) or more like an $H_{\infty}$ filter with more emphasis on sensor noise attenuation (larger $\gamma$ and $V$ ).

Remark 2. It should be noted that. a Beard-Jones fault detection filter can detect all of the $\mu_{j}$ 's. The filter that we propose here can detect only one fault at a time.

Remark 3. Lee and Gilson derive a filter for fault detection via linear quadratic methods in [7]. They do not discuss their work in relation to fault detection filters, but in many respects their final result is similar to the game theoretic filter described here. Moreover, they also apply their work to the same example that we examine in the next, section with similar results. 
In Section 2 we noted that molsservalbility subspaces are used in current. fault detection filter design methods because they allow the designer to specify (within complex conjugate symmetry) all of the eigenvalues of the filter. Such design freedom exists with these sulsppaces because they include any invariant zero directions which arise out of the triple $\left(C, A, \dot{F}_{1}\right)$.

Invariant zeros [20] are those complex numbers $\lambda$ such that the system mat.rix:

$$
P(\lambda)=\left[\begin{array}{cc}
A-\lambda I & \hat{F}_{1} \\
C & 0
\end{array}\right]
$$

loses rank. Invariant zero directions $x$ are those complex vectors such that:

$$
\left[\begin{array}{cc}
A-\lambda I & \hat{F}_{1} \\
C & 0
\end{array}\right]\left\{\begin{array}{l}
x \\
w
\end{array}\right\}=0
$$

Invariant zeros are a concern in fault detection filter design because they cannot. be eliminated by output injection feedback, i.e. an estimator. Moreover, Douglas (Proposition 2.9 [4]) has shown that if we do not include invariant zero directions in a $(C, A)$-invariant subspace, the corresponding invariant zero will show up as an eigenvalue in the factor space when we try to design an estimator for the system.

In the next theorem, we will show that the game theoretic filter places invariant zeros in the kernal of II. Since we have already shown that ker $\Pi$ is a $(C, A)$-invariant subspace, this theorem then implies that ker $\Pi$ is an unobservability subspace. For our purposes, this result is not as important as it is for spectral design methods since pole locations fall out. from the game solution. It. does, lowever, connect this filter to existing detection filters by showing that. the invariant subspace formed by the game theoretic approach is the same kind cliosen in standard detection filter desigu algorithms.

The proof to the upcoming theorem is based on the fact that positive semi-definite, symmetric matrices such as $\Pi$ always have non-singular, transformations - say $\Gamma$ - that are orthonormal $\left(\Gamma^{T} \Gamma=I\right)$ and that convert the matrix into the form:

$$
\Gamma \Pi \Gamma^{T}=\left[\begin{array}{ll}
\bar{\Pi} & 0 \\
0 & 0
\end{array}\right]
$$

where $\bar{\Pi}$ is positive definite. Define:

$$
C \Gamma^{T}=\left[\begin{array}{ll}
C_{1} & C_{2}
\end{array}\right] \quad \Gamma A \Gamma^{T}=\left[\begin{array}{ll}
A_{11} & A_{12} \\
A_{21} & A_{22}
\end{array}\right] \quad \Gamma \dot{F}_{1}=\left[\begin{array}{l}
F_{11} \\
F_{12}
\end{array}\right]
$$

Because $\Pi \dot{F}_{1}=0$ implies $\Gamma \Pi \dot{F}_{1}=0$, we can inmediately conclucle that: 


$$
\Gamma \Pi \Gamma^{T} \Gamma \dot{F}_{1}=\left[\begin{array}{ll}
\bar{\Pi} & 0 \\
0 & 0
\end{array}\right]\left[\begin{array}{l}
F_{11} \\
F_{22}
\end{array}\right]=\bar{\Pi} F_{11}=0
$$

Which, since $\bar{\Pi}$ is positive-definite, implies:

$$
F_{11}=0
$$

Theorem 5.3. The invariant zeros of $\left(C, A, \hat{F}_{1}\right)$ lie in ker $\Pi$

Proof.

Transform the system matrix $P(\lambda)$ using the transformation matrix, $\Gamma$ described by $(79)$ :

$$
\left[\begin{array}{ll}
\Gamma & 0 \\
0 & I
\end{array}\right]\left[\begin{array}{cc}
A-\lambda I & \hat{F}_{1} \\
C & 0
\end{array}\right]\left[\begin{array}{cc}
\Gamma^{T} & 0 \\
0 & I
\end{array}\right]=\left[\begin{array}{lll}
A_{11}-\lambda I & A_{12} & 0 \\
A_{21} & A_{22}-\lambda I & F_{12} \\
C_{1} & C_{2} & 0
\end{array}\right]
$$

(80) clearly shows that if invariant zeros exist they can only arise from the triple $\left(C_{2}, A_{22}, F_{12}\right)$ which corresponds to the subspace determined by ker II. In fact, (80) shows that the only kind of zeros which coild occur in the range of II are output decoupling zeros [20] arising from values of $\lambda$ which make:

$$
\left[\begin{array}{l}
A_{11}-\lambda I \\
A_{21} \\
C_{1}
\end{array}\right]
$$

lose rank. However, since we liave assumed that. $(C, A)$ is observable, these zeros cannot occur.

\section{Fault Detection with the Limiting Form of the Game Theoretic Filter}

In this section, we will show that a reduced-order fault detector can be derived from the limiting form of the game theoretic filter. The results from this section are more easily applied to time-invariant systems, but we will give an overview of how to apply these results to time-varying systems. For the moment, let. us restrict, ourselves the LTI systems. Using the transformation, $\Gamma$, defined hy $(79)$, set:

$$
i=\left\{\begin{array}{l}
\hat{\eta}_{1} \\
\hat{\eta}_{2}
\end{array}\right\}=\Gamma \dot{x}
$$

Pre-multiply (45) by $\Gamma$ and make use of the identity $\Gamma^{T} \Gamma=I$ to get:

$$
\left.\left(\Gamma \Pi \Gamma^{T}\right) \dot{i}=\left(\Gamma \Pi \Gamma^{T}\right)\left(\Gamma A \Gamma^{T}\right) \dot{\eta}+\Gamma C^{T} \bar{V}^{-1}\left(y-C \Gamma^{T} \dot{\eta}\right)\right)
$$


The transformed filter equation (81) is sen to be:

$$
\left[\begin{array}{cc}
\bar{\Pi} & 0 \\
0 & 0
\end{array}\right]\left\{\begin{array}{l}
\dot{\eta}_{1} \\
\dot{\eta}_{2}
\end{array}\right\}=\left[\begin{array}{cc}
\bar{\Pi} & 0 \\
0 & 0
\end{array}\right]\left[\begin{array}{ll}
A_{11} & A_{12} \\
A_{21} & A_{22}
\end{array}\right]\left\{\begin{array}{l}
\dot{\eta}_{1} \\
\dot{\eta}_{2}
\end{array}\right\}+\left\{\begin{array}{l}
C_{1}^{T} \\
C_{2}^{T}
\end{array}\right\} \bar{V}^{-1}\left(y-\left[C_{1} C_{2}\right]\left\{\begin{array}{l}
\dot{\eta}_{1} \\
\dot{\eta}_{2}
\end{array}\right\}\right)
$$

From (82) we grel a dyuamic equation for $i_{1}$ :

$$
\bar{\Pi} \dot{\hat{\eta}}_{1}=\bar{\Pi} A_{11} \hat{\eta}_{1}+\bar{\Pi} A_{12} \hat{\eta}_{2}+C_{1}^{T} \bar{V}^{-1}\left(y-C_{1} \dot{\eta}_{1}-C_{2} \dot{\eta}_{2}\right)
$$

and a static equation for $\hat{\eta}_{2}$ :

$$
\hat{\eta}_{2}=\left(C_{2}^{T} \bar{V}^{-1} C_{2}\right)^{-1} C_{2}^{T} \bar{V}^{-1}\left(y-C_{1} \hat{\eta}_{1}\right)
$$

Define

$$
K:=\left(C_{2}^{T} \bar{V}^{-1} C_{2}\right)^{-1} C_{2}^{T} \bar{V}^{-1}
$$

so that the substitution of $(85)$ and $(84)$ into $(83)$ gives us an estimator for $\hat{\eta}_{1}$ :

$$
\dot{\hat{\eta}}_{1}=A_{11} \dot{\eta}_{1}+\left[\bar{\Pi}^{-1} C_{1}^{r} \bar{V}^{-1}\left(I-C_{2} K\right)+A_{12} K\right]\left(y-C_{1} \dot{\eta}_{1}\right)
$$

To see that the reduced-order estimator $(86)$ is unaffected by the nuisance fault $\hat{\mu}_{2}$, we will derive the error equation for the reduced-order filter. Define:

$$
\eta=\left\{\begin{array}{l}
\eta_{1} \\
\eta_{2}
\end{array}\right\}:=\Gamma x \quad e_{1}:=\hat{\eta}_{1}-\eta_{1} \quad e_{2}:=\hat{\eta}_{2}-\eta_{2}
$$

We begin by premultiplying the dynamical equation (19) by the Riccati Matrix, $\Pi$. Since $\Pi \hat{F}_{1}=0$, we get:

$$
\Pi \dot{\boldsymbol{x}}=\Pi A \boldsymbol{x}
$$

This can be pre-nultiplied by $\Gamma$ and manipulated into:

$$
\left[\begin{array}{cc}
\bar{\Pi} & 0 \\
0 & 0
\end{array}\right]\left\{\begin{array}{l}
\dot{\eta}_{1} \\
\dot{\eta}_{22}
\end{array}\right\}=\left[\begin{array}{cc}
\bar{\Pi} & 0 \\
0 & 0
\end{array}\right]\left[\begin{array}{ll}
A_{11} & A_{12} \\
A_{21} & A_{22}
\end{array}\right]\left\{\begin{array}{l}
\eta_{1} \\
\eta_{2}
\end{array}\right\}
$$

As with the estimator equation, (87) shows that only a portion of the st.ate-space possesses dynamics:

$$
\bar{\Pi} \dot{\eta}_{1}=\bar{\Pi} A_{11} \eta_{1}+\bar{\Pi} A_{12} \eta_{2}
$$

Using (88) to get. an error equation would leave terms in $\eta_{2}$ or $\epsilon_{2}$. In anticipation of this, we transform the measurement. equation:

$$
y=C x+v=C \Gamma^{T} \Gamma x+v=C_{1} \eta_{1}+C_{1} y_{2}+v
$$


and use (89) and (84) to solve for $\epsilon_{2}$ :

$$
c_{2}=\left(C_{2}^{T} \bar{V}^{-1} C_{2}\right)^{-1}\left(C_{2}^{T} \bar{V}^{-1} C_{1} c_{1}+C_{2}^{T} \bar{V}^{-1} v\right)=K\left(C_{1} c_{1}-v\right)
$$

Subtract (88) from (83) and sulstitiute (89) for $y$ :

$$
\bar{\Pi} \dot{\varepsilon}_{1}=\bar{\Pi} A_{11} \epsilon_{1}+\bar{\Pi} A_{12} \varepsilon_{2}+C_{1}^{T} \bar{V}^{-1} C_{1} e_{1}+C_{1}^{T} \bar{V}^{-1} C_{2} e_{2}+C^{T} \bar{V}^{-1} v
$$

Using (90) and collecting terms, we can turn the previous equation into:

$$
\dot{e}_{1}=\left[A_{11}-\bar{\Pi}^{-1} C_{1}^{T} \bar{V}^{-1}\left(I-C_{2} K\right) C_{1}-A_{12} K C_{1}\right] e_{1}+\left[\bar{\Pi}^{-1} C_{1}^{T} \bar{V}^{-1}\left(I-C_{2} K\right)+A_{12} K\right] v
$$

Note that nuisance fault, $\hat{i}_{2}$, appears nowhere in the estimator (86) nor in the error equation (91). Thus, in the limit, we get a reduced-order estimator completely uninfluenced by the nuisance faults. The term $\left(C_{2}^{T} \bar{V}^{-1} C_{2}\right)^{-1}$ appears in various places in the reduced-order estimator. This inverse will always exist since $\bar{V}$ is positive definite and since the assumption of $(C, A)$ observalility guarantees that $C_{2}$ will have full column rank.

Remark 4. The reduced-order filter derived here is similar to the residual generator derived by Massoumnia, et al. in [3]. An important difference, however, is that Massoumnia begins his design process by factoring out the reachable space of the nuisance faults. As a result, he has the freedom to use any kind of filter design technique for the lower dimensional state-space. The trade-off, however, is that the system reduction in Massoumnia's filter is sensitive to the inexactness of the plant, model. Variations in the plant. will change the reachable subspace and may, as a result, degrade the performance of the recluced-order detector. In the game filter, the order reduction comes at the end of the design process. Thus, there is no design freedom left to tune the reduced-order filter, but the game formulation used to obtain the filter makes it possible to account for model uncertainties. Thus, it can be argued that the order reduction used here is more robust.

The Goh transformation and corresponding Riccati equation greatly extend our ability to analyze the reducedorder estimator. In fact with the Gol, Riccati equation we can show that there always exist a stabilizing solution for the reduced order estimator. Applying the transformation $\Gamma$ to (74), we get:

$$
\begin{array}{r}
-\Gamma \dot{S} \Gamma^{T}=\Gamma S \Gamma^{T} \Gamma A \Gamma^{T}+\Gamma A^{T} \Gamma^{T} \Gamma S \Gamma^{T}+\Gamma C^{T}\left(\dot{H}_{1}^{T} Q_{1} \dot{H}_{1}-\bar{V}^{-1}\right) C \Gamma^{T} \\
+\left[\Gamma S \Gamma^{T}\left(\Gamma A \Gamma^{T} \Gamma B_{i}-\Gamma \dot{B}_{i}\right)-\Gamma C^{T} \bar{V}^{-1} C \Gamma^{T} \Gamma B_{i}\right]\left(B_{i}^{T} C^{T} \bar{V}^{-1} C B_{i}\right)^{-1} \\
\times\left[\left(\Gamma A \Gamma^{T} \Gamma B_{i}-\Gamma \dot{B}_{i}\right)^{T} \Gamma S \Gamma^{T}-\Gamma C^{T} \bar{V}^{-1} C \Gamma^{T} \Gamma B_{i}\right]
\end{array}
$$


Define:

$$
\Gamma B_{i}=\left[\begin{array}{c}
B_{11} \\
B_{12}
\end{array}\right]
$$

As in section 5, the necessary condition $S B_{i}=0$ will lead to $B_{11}=0$ since $\Gamma S \Gamma^{T} \Gamma B_{i}=0 \Rightarrow \bar{S} B_{11}=0$ and $\bar{S}$ is positive-definite. Also, if we carry the transformation through, a number of terms fall out because the projector $\dot{H}_{1}$ has been constructed so that:

$$
\hat{H}_{1} C B_{\mathrm{i}}=0 \Longrightarrow \hat{H}_{1} C \Gamma^{T} \Gamma B_{i}=0 \Longrightarrow\left[\begin{array}{ll}
\hat{H}_{1} C_{1} & \hat{H}_{1} C_{2}
\end{array}\right]\left[\begin{array}{c}
0 \\
B_{12}
\end{array}\right]=0 \Rightarrow \hat{H}_{1} C_{2} B_{12}=0
$$

We we show later that $B_{i}$ can always be augmented so that $B_{12}$ is an invertible square matrix. Hence (93) impiies:

$$
\hat{H}_{1} C_{2}=0
$$

Using (94) and working through all of the transformations leads to:

$$
\begin{array}{r}
\left.\left[\begin{array}{cc}
-\dot{\bar{S}} & 0 \\
0 & 0
\end{array}\right]=\left[\begin{array}{cc}
\bar{S} A_{11} & \bar{S} A_{12} \\
0 & 0
\end{array}\right]+\left[\begin{array}{cc}
A_{11}^{T} \bar{S} & 0 \\
A_{1,}^{T} \bar{S} & 0
\end{array}\right]+\left(\begin{array}{rr}
\bar{S} A_{12} B_{12}-\bar{S} \dot{B}_{11} \\
0
\end{array}\right]-\left[\begin{array}{c}
C_{1}^{T} \bar{V}^{-1} C_{2} B_{12} \\
C_{2}^{T} \bar{V}^{1} C_{2} B_{12}
\end{array}\right]\right) \\
\times\left(B_{12}^{T} C_{2}^{T} \bar{V}^{-1} C_{2} B_{12}\right)^{-1}\left(\left[\begin{array}{ll}
B_{12}^{T} A_{12} \bar{S}-\dot{B}_{11} \bar{S} & 0
\end{array}\right]-\left[\begin{array}{cc}
B_{12}^{T} C_{2}^{T} \bar{V}^{-1} C_{1}^{T} & B_{12}^{T} C_{2}^{T} \bar{V}^{-1} C_{2}^{T}
\end{array}\right]\right) \\
+\left[\begin{array}{rr}
C_{1}^{T}\left(\hat{H}_{1}^{T} Q_{1} \hat{H}_{1}-\bar{V}^{-1}\right) C_{1} & -C_{1}^{T} \bar{V}^{-1} C_{2} \\
-C_{2}^{T} \bar{V} C_{1} & -C_{2}^{T} \bar{V}^{-1} C_{2}
\end{array}\right]
\end{array}
$$

From (95) we get three equations:

$$
\begin{array}{r}
-\dot{\bar{S}}=C_{1}^{T}\left(\hat{H}_{1}^{T} Q_{1} \hat{H}_{1}-\bar{V}^{-1}\right) C_{1}+\bar{S} A_{11}+A_{11}^{T} \bar{S}+\left(\bar{S} A_{12} B_{12}-\bar{S} \dot{B}_{11}-C_{1}^{T} \bar{V}^{-1} C_{2,} B_{12}\right)\left(B_{12}^{T} C_{2}^{T} \bar{V}^{-1} C_{2} B_{12}\right)^{-1} \\
\times\left(\bar{S} A_{12} B_{12}-\bar{S} \dot{B}_{11}-C_{1}^{T} \bar{V}^{-1} C_{2} B_{12}\right)^{T} \\
0=-C_{1}^{T} \bar{V}^{-1} C_{2}+\bar{S} A_{12}-\left(\bar{S} A_{1 !} B_{12}-\bar{S} \dot{B}_{11}-C_{1}^{T} \bar{V}^{-1} C_{2}^{T} B_{12}\right)\left(B_{12}^{T} C_{12}^{T} \bar{V}^{-1} C_{2,} B_{12}\right)^{-1} B_{12}^{T} C_{2}^{T} \bar{V}^{-1} C_{2} \\
0=-C_{2}^{T} \bar{V}^{-1} C_{2}+C_{2}^{T} \bar{V}^{-1} C_{2} B_{12}\left(B_{12}^{T} C_{2}^{T} \bar{V}^{-1} C_{2} B_{12}\right)^{-1} B_{12}^{T} C_{2}^{T} \bar{V}^{-1} C_{2} .
\end{array}
$$

However, if we post-multiply $(98)$ by $B_{12}$ and cancel terms we obtain the identity $0=0$. If we post-multiply $(97)$ by $B_{12}$ we obtain:

$$
0=\bar{S} \dot{B}_{11} \Rightarrow \dot{B}_{11}=0 \text {. }
$$

Thus, we need only (96), which thanks to (99) can he simplified to: 


$$
\begin{array}{r}
-\dot{\bar{S}}=C_{1}^{T}\left(\dot{H}_{1}^{T} Q_{1} H_{1}-\bar{V}^{-1}\right) C_{1}+\bar{S} A_{11}+A_{11}^{T} \bar{S}+\left(\bar{S} A_{12} B_{12}-C_{1}^{T} \bar{V}^{-1} C_{2} B_{12}\right) \\
\times\left(B_{12}^{T} C_{2}^{T} \bar{V}^{-1} C_{2} B_{12}\right)^{-1}\left(\bar{S} A_{12} B_{12}-C_{1}^{T} \bar{V}^{-1} C_{2} B_{12}\right)^{T}
\end{array}
$$

Now if $i=1$, then $B_{i}=\hat{F}_{1}$ and the rank of $\hat{F}_{1}$ equals the dimension of the kernal of $S . B_{12}=F_{12}$ will then be square and, moreover, it. will be invertible since $\hat{F}_{1}$ was assumed monic. Given this, we can simplify $(100)$ to:

$$
\begin{array}{r}
-\dot{\bar{S}}=C_{1}^{T}\left(\hat{H}_{1}^{T} Q_{1} \hat{H}_{1}-\bar{V}^{-1}\right) C_{1}+\bar{S} A_{11}+A_{11}^{T} \bar{S}+\left(\bar{S} A_{12}-C_{1}^{T} \bar{V}^{-1} C_{2}\right)\left(C_{2}^{T} \bar{V}^{-1} C_{2}\right)^{-1}\left(\bar{S} A_{12}-C_{1}^{T} \bar{V}^{-1} C_{2}\right)^{T} \\
\bar{S}\left(t_{0}\right)=0
\end{array}
$$

where the boundary condition comes from (68). This leads us to the key result of this section.

Theorem 6.1. The solution $\bar{S}$ to (101) gives a stabilizing solution for the reduced-order estimator (86).

Proof. Using the same transformation to derive both (101) and (86) will ensure that $\bar{S}$ is of proper dimension for (86). Substitute $\bar{S}$ into (\$6) directly for $\bar{\Pi}$. The resulting estimator is:

$$
\dot{\hat{\eta}}_{1}=\left(A_{11}-\left[\bar{S}^{-1} C_{1}^{T} \bar{V}^{-1}\left(I-C_{2} K\right)+A_{12} K\right] C_{1}\right) \hat{\eta}_{1}+\left[\bar{S}^{-1} C_{1}^{T} \bar{V}^{-1}\left(I-C_{2} K\right)+A_{12} K\right] y
$$

where $K:=\left(C_{2}^{T} \bar{V}^{-1} C_{2}\right)^{-1} C_{2}^{T} \bar{V}^{-1}$. Clearly, the stability of the estimator depends upon the closed-loop state matrix, $\left(A_{11}-\left[\bar{S}^{-1} C_{1}^{T} \bar{V}^{-1}\left(I-C_{2} K\right)+A_{12} K\right] C_{1}\right)$. Now, if we go back to (101), multiply out the quadratic, and use the definition for $K$, we get:

$$
-\dot{\bar{S}}=\bar{S}\left(A_{11}-A_{12} K C_{1}\right)+\left(A_{11}-A_{12} K C_{1}\right)^{T} \bar{S}+C_{1}^{T}\left[\hat{H}_{1}^{T} Q_{1} \dot{H}_{1}-\bar{V}^{-1}\left(I-C_{2} K\right)\right] C_{1}+\bar{S} A_{12}\left(C_{2}^{T} \bar{V}^{-1} C_{2}\right)^{-1} A_{12}^{T} \bar{S}
$$

If we add and subtract. $C_{1}^{T} \bar{V}^{-1}\left(I-C_{2} K\right) C_{1}$ too (104) and rearrange terms we get:

$$
\begin{array}{r}
-\dot{\bar{S}}=\bar{S}\left(A_{11}-A_{12} K C_{1}-\bar{S}^{-1} C_{1}^{T} \bar{V}^{-1}\left(I-C_{2} K\right) C_{1}\right)+\left(A_{11}-A_{12} K C_{1}-\bar{S}^{-1} C_{1}^{T} \bar{V}^{-1}\left(I-C_{2} K\right) C_{1}\right)^{T} \bar{S} \\
\left.+C_{1}^{T}\left[\hat{H}_{1}^{T} Q_{1} \hat{H}_{1}+\bar{V}^{-1}\left(I-C_{2} K\right)\right] C_{1}+\bar{S} A_{12}\left(C_{2}^{T} \bar{V}^{-1} C_{2}\right)^{-1} A_{12}^{T} \bar{S}\right] C_{1}
\end{array}
$$

Note that $C_{1}^{T} \bar{V}^{-1}\left(I-C_{2} K\right) C_{1}$ is symmetric. (105) implies:

$$
\dot{\bar{S}}+\bar{S}\left(A_{11}-A_{12} K C_{1}-\bar{S}^{-1} C_{1}^{T} \bar{T}^{-1}\left(I-C_{2} K\right) C_{1}\right)+\left(A_{11}-A_{12} K C_{1}-\bar{S}^{-1} C_{1}^{T} \bar{V}^{-1}\left(I-C_{2} K\right) C_{1}\right)^{T} \bar{S} \leq 0
$$

which by Lyapunov's Direct. Method [21] implies that. $\left(A_{11}-A_{12} K C_{1}-\bar{S}^{-1} C_{1}^{T} \bar{V}^{-1}\left(I-C_{2} K\right) C_{1}\right)$ is stable. For time-invariant systems, this implies that the closed-loop eigenvalues lie in the open left-half plane. 
What happens, however, when $i>1$ and dim(kers) $>\operatorname{rank} B_{i}$ ? The matrix $B_{12}$ will no longer be suluare and the reduced-order Riccati Equation will be stuck in the form of Equation 100 which is not the same as what is needed in the proof for stability (Equation 101). It would thus seem that we cannot guarantee stability in the general case.

It turns out, however, that by angmenting the failure map in the original problem statement, we can always convert the reduced-order Riccati equation into the desired form (101). The necessary augmentation turns out to be:

$$
\bar{F}_{1}=\left[\begin{array}{llll}
B_{i} & B_{i-1} & \ldots & B_{1}
\end{array}\right]
$$

The new game problem for the limiting case is:

$$
\begin{aligned}
& \min _{\bar{x}} \max _{\bar{H}_{2}} J^{*}=\int_{t_{0}}^{t_{1}}\left[\|x-\hat{x}\|_{C^{T}}^{2} \dot{H}_{1}^{T} Q_{2} \dot{H}_{1} C+(x-\hat{x})^{T} C^{T} \hat{H}_{1}^{T} Q_{1} \hat{H}_{1} C \bar{F}_{1} \bar{H}_{2}+\left\|\bar{\mu}_{2}\right\|_{\bar{F}_{1}^{T}}^{2} C^{T} \dot{H}_{1}^{T} Q_{1} \dot{H}_{1} C \bar{F}_{2}\right. \\
& -\|y-C x\|_{\bar{V}^{-1}}^{2}-(y-C x)^{T} \bar{V}^{-1} C \bar{F}_{1} \bar{\mu}_{2}-\bar{\mu}_{2}^{T} \bar{F}_{1}^{T} C^{T} \bar{V}^{-1}(y-C x)-\left\|\bar{\mu}_{2}\right\|_{\bar{F}_{1}^{T} C^{T} \bar{V}^{-1} c \bar{F}_{1}}^{j} d t
\end{aligned}
$$

subject to:

$$
j=A x+\bar{F}_{1} \bar{\mu}_{2}
$$

where $\bar{\beta}_{2}$ is the augmented failure signal which has many inputs as there are columns in $\bar{F}_{1}$. Note, that here we have gone back to the pre-transformed problem (where the state is $x$, not $\alpha_{i}$ ). We will show that this new problem leads to a Riccati equation which is equivalent to (74). In this equation, however, the reduced-order version is easily seen to reduce to the desired form (101). The equivalence of the two equations then implies that the same reduced form holds for both.

The augmented failure map, $\bar{F}_{1}$ is such that $C \bar{F}_{1} \neq 0$, thereby stopping the transformation process after one iteration. The solution to this game leads to a Goh Riccati Equation:

$$
\begin{array}{r}
-\dot{S}=S A+A^{T} S+C^{T}\left(\hat{H}_{1}^{T} Q_{1} \dot{H}_{1}-\bar{V}^{-1}\right) C+\left[S\left(A \bar{F}_{1}-\dot{\bar{F}}_{1}\right)-C^{T} \bar{V}^{-1} C \bar{F}_{1}\right] \\
\times\left(\bar{F}_{1}^{T} C^{T} \bar{V}^{-1} C \bar{F}_{1}\right)^{-1}\left[\left(A \bar{F}_{1}-\dot{\bar{F}}_{1}\right)^{T} S-\bar{F}_{1}^{T} C^{T} \bar{V}^{-1} C\right]
\end{array}
$$

with a boundary condition given hy $(68)$. The solution, $S$, to $(110)$ is such that $\operatorname{dim}(\operatorname{ker} \bar{S})=\operatorname{rank} \bar{F}_{1}$. Hence, after the transformation and defining:

$$
\left[\begin{array}{l}
\bar{F}_{11} \\
\bar{F}_{12}
\end{array}\right]=\Gamma \bar{F}_{1}
$$


the reduced-order Riccati Equation:

$$
\begin{array}{r}
-\dot{\bar{S}}=C_{1}^{T}\left(\dot{H}_{1}^{T} Q_{1} \bar{H}_{1}-\bar{V}^{-1}\right) C_{1}+\bar{S} A_{11}+A_{11}^{T} \bar{S}+\left(\bar{S} A_{12} \bar{F}_{12}-C_{1}^{T} \bar{V}^{-1} C_{2} \bar{F}_{12}\right) \\
\times\left(\bar{F}_{12}^{T} C_{2}^{T} \bar{V}^{-1} C_{2} \bar{F}_{12}\right)^{-1}\left(\bar{S} A_{12} \bar{F}_{12}-C_{1}^{T} \bar{V}^{-1} C_{2} \bar{F}_{12}\right)^{T} .
\end{array}
$$

can be simplified to (101) because $\bar{F}_{12}$ is square and invertible. We know that $\bar{F}_{12}$ is square and invertible because the construction of $\bar{F}_{1}$ ensures that $\bar{F}_{1}$ has full column rank and that the size of ker $S$, which determines the order reduction, is equal to this column rank.

Proposition 6.2. The Golı Riccati Equation of the augmented system (110) is equivalent to the Goh Riccaii Equation of the original system (74).

Proof. It is immediate that

$$
C \bar{F}_{1}=C\left[\begin{array}{llll}
B_{i} & B_{i-1} & \ldots & B_{1}
\end{array}\right]=C B_{i}
$$

If we examine the term $S A \bar{F}_{i}-\dot{\bar{F}}_{1}$ in $(110)$ :

$$
\begin{aligned}
S\left(A \bar{F}_{1}-\dot{\bar{F}}_{1}\right) & =S A\left[\begin{array}{llll}
B_{i} & B_{i-1} & \ldots & B_{1}
\end{array}\right]+S\left[\begin{array}{lll}
\dot{B}_{i} & \dot{B}_{i-1} & \ldots
\end{array}\right] \\
& =\left[\begin{array}{lll}
S A B_{i}-S \dot{B}_{i}, S A B_{i-1}-S \dot{B}_{i}, \ldots, S A B_{1}-S \dot{B}_{1}
\end{array}\right] \\
& =\left[\begin{array}{lll}
S A B_{i}-S \dot{B}_{i}, S B_{i}, S B_{i-1}, \ldots, S B_{2}
\end{array}\right]
\end{aligned}
$$

Because of Proposition 4.2, this simplifies to

$$
S\left(A \bar{F}_{1}-\dot{\bar{F}}_{1}\right)=S\left(A B_{i}-\dot{B}_{i}\right)
$$

Given, (112) and (113), the Gol Riccati Expation for the augmented system (110) simplifies to (74).

Reduced-order filters for the time-varying are much harder to come by since the transformation matrix, $\Gamma$, will now be a function of time. In this case, the only likely option left to the analyst is to use the results of [22] which give differential equations for the eigenvertors and eigenvalues of the solution to a time-varying Riccati Equation. From here the reduced-order Riccali Matrix, the transformed system equation, and finally the reduced order filter can be formed through a transformation matrix based upon the eigenvectors. Needless to say, the computation required here will be quite intensive. The stat.e and measurement. mat.rices will also have to be transformed at each time step and only then can the filter be formed and propagated. The point here is that it is possible to find a reduced filter 
for the time-varying case, though the effort may ontweiglt the benefits. Since the full-order filter is always available, this is not a serious problem.

The analyst. has many options when designing a game theoretic filter. In the case of the full-order filter he has the freedom to choose the different weighting matrices and $\gamma$. For reduced-order filters, he can use either the solution to the Goh Riccati Equation (74) or the solution of linear matrix inecjuality (56) with $\gamma=0$ to find the needed transformation matrix and reduced-order filter gain. He also has the reduced-order Riccati Equation (101). Moreover, he can mix the two approaches (e.g. using the LMI to find the transformation matrix and using the reduced-order Goh Riccati Equation to find the gain). This flexibility is important, because the solution to the Goh equations may be ill-conditioned when several iterations of the Goh Transformation are needed to generate the Riccati Equation. The appearance of powers of $A$ in the resulting equation may cause problems with the numerical solution. Thus, though the Riccati solution represents the saddle-point solution, it may not be the best choice from numerical considerations.

\section{Example: Accelerometer Fault Detection for the F16XL}

\subsection{Problem Statement}

To demonstrate the effectiveness of the game theoretic filter, the F16XL example of [5] is re-examined. The aircraft dynamics are linearized about. trimmed level fight. at 10,000 ft. altitude and Mach 0.9 . For simplicity, a reduced-order, five-state model of the longitudinal dynamics (including a first-order wind gust. model) is considered:

$$
\begin{gathered}
\dot{x}=A x+B w_{w g} \\
y=C x+v .
\end{gathered}
$$

The five components of the states are:

$$
x=\left\{\begin{array}{c}
u \\
u \\
y \\
\theta \\
u_{y}
\end{array}\right\} \begin{aligned}
& \text { long. velocity }(\mathrm{ft} / \mathrm{sec}) \\
& \text { normal vclocity }(\mathrm{ft} / \mathrm{sec}) \\
& \text { pitch rate }(\mathrm{deg} / \mathrm{sec}) \\
& \text { pitch }(\mathrm{deg}) \\
& \text { wirul gust }(\mathrm{ft} / \mathrm{sec})
\end{aligned}
$$

The measurements are:

$$
y=\left\{\begin{array}{c}
q \\
\theta \\
A_{z} \\
A_{x}
\end{array}\right\} \begin{aligned}
& \text { pitch ratc (deg/ } / \mathrm{sec}) \\
& \text { pitch (deg) } \\
& \text { long. accelcration }\left(\mathrm{ft} / \mathrm{sec}^{2}\right) \\
& \text { normal accclcration }\left(\mathrm{ft} / \mathrm{sec}^{2}\right)
\end{aligned}
$$

The input, $w_{w g}$, is windgust. and $v$ is the sensor noise. $v$ is also assumed to be weighted by $V=\nu I_{4}$. The resulting system matrices are: 


$$
\begin{gathered}
A=\left[\begin{array}{ccccc}
-.0674 & .0430 & -.8886 & -.5587 & .0430 \\
.0205 & -1.4666 & 16.5800 & -.0299 & -1.4666 \\
.1377 & -1.6788 & -.6819 & 0 & -1.6788 \\
0 & 0 & 1 & 0 & 0 \\
0 & 0 & 0 & 0 & -1.1948
\end{array}\right] \\
B^{T}=\left[\begin{array}{llllll}
0 & 0 & 0 & 0 & 2.0156
\end{array}\right] \\
C=\left[\begin{array}{ccccc}
0 & 0 & 1 & 0 & 0 \\
0 & 0 & 0 & 1 & 0 \\
0.0139 & 1.0517 & 0.1485 & -0.0299 & 0 \\
-0.0677 & 0.0431 & 0.0171 & 0 & 0
\end{array}\right]
\end{gathered}
$$

It is desired to detect a normal accelerometer fault, $A_{2}$, in the presence of the wind gust disturbance and the sensor noise. Following the modelling techniques described in section 2, we incorporate the accelerometer faults into the system by modifying our st.ate equations to:

$$
\begin{aligned}
& \dot{x}=A x+B w_{w g} \\
& y=C x+E_{A_{z} \mu_{A_{s}}+v .}
\end{aligned}
$$

where $E_{A_{t}}=\left[\begin{array}{llll}0 & 0 & 1 & 0\end{array}\right]^{T}$ and $\mu_{A_{t}}$ is the acceleromter failure signal. We then convert (119) and (120) into:

$$
\begin{aligned}
& \dot{x}=A x+F_{A_{1} \mu_{A_{2}}}+F_{w g} \mu_{w g} \\
& y=C x+v_{1}
\end{aligned}
$$

$F_{w g}$ is simply $B_{u, g} ; \mu_{u v g}$ is the wind gust, $w_{u, g}$. The failure map, $F_{A_{z}}$ and the failure signal $\mu_{A_{z}}$ are:

$$
F_{A_{t}}=\left[\begin{array}{cc}
0.6003 & 0 \\
0.9429 & -1.3706 \\
0 & -1.5003 \\
0 & 0 \\
0 & 0
\end{array}\right] \quad \mu_{A_{1}}=\left\{\begin{array}{l}
\mu_{1} \\
\mu_{2}
\end{array}\right\}
$$

Finally, the natural projections on the filter residual are:

$$
\begin{gathered}
\hat{H}_{1}=I-\left(C A F_{w, q}\right)\left[\left(C A F_{w, q}\right)^{T}\left(C A F_{w, q}\right)\right]^{-1}\left(C A F_{w, q}\right)^{T}=\left[\begin{array}{cccc}
0.5330 & 0 & -0.4982 & -0.0264 \\
0 & 1 & 0 & 0 \\
-0.4982 & 0 & 0.4685 & -0.0281 \\
-0.0264 & 0 & -0.0281 & 0.9985
\end{array}\right] \\
N_{1}=I-\hat{H}_{1}=\left(C A F_{w, q}\right)\left[\left(C A F_{w, q}\right)^{T}\left(C A F_{w, q}\right)\right]^{-1}\left(C A F_{w, q}\right)^{T}=\left[\begin{array}{cccc}
0.4670 & 0 & 0.4982 & 0.0264 \\
0 & 0 & 0 & 0 \\
0.4982 & 0 & 0.5315 & 0.0281 \\
0.0264 & 0 & 0.0281 & 0.0015
\end{array}\right] .
\end{gathered}
$$




\subsection{Full-Order Filter Design}

Equation 34, the Riccati Equation in terms of $P$, was used for this example, though we could have just as easily used (31), the Riccati Equation in terms of $\Pi$. To bring sensor noise weighting, $V(=\nu I)$, to zero with the disturbance bound, it is assumed that $\nu$ is some multiple of $\gamma$. By t.rial and error, it. was found that:

$$
\nu=5 \times 10^{-8}, \quad \frac{\nu}{\gamma}=2, \quad Q_{1}=R_{1}=M_{2}=I
$$

gave the results seen in Figure 1. For the given parameters above, the solution of (34) is:

$$
P=10^{-6} \times\left[\begin{array}{ccccc}
.019161 & -.25947 & -.20981 & -.00448 & 11.059 \\
-.25947 & 7.5971 & 7.1863 & .076754 & -376.33 \\
-.20981 & 7.1863 & 7.9932 & .011879 & -428.83 \\
-.00448 & .076754 & .011879 & .00397 & -.098976 \\
11.069 & -376.33 & -428.83 & -.098976 & 45255
\end{array}\right]
$$

resulting in a gain:

$$
L=\left[\begin{array}{cccc}
-2.0981 & -0.0448 & -3.0364 & -0.16068 \\
71.863 & 0.7675 & 90.511 & 4.679 \\
79.932 & 0.1188 & 87.416 & 4.6062 \\
0.1188 & 0.0398 & 0.8230 & 0.0381 \\
-4288.3 & -0.9897 & -4593.1 & -243.02
\end{array}\right]
$$

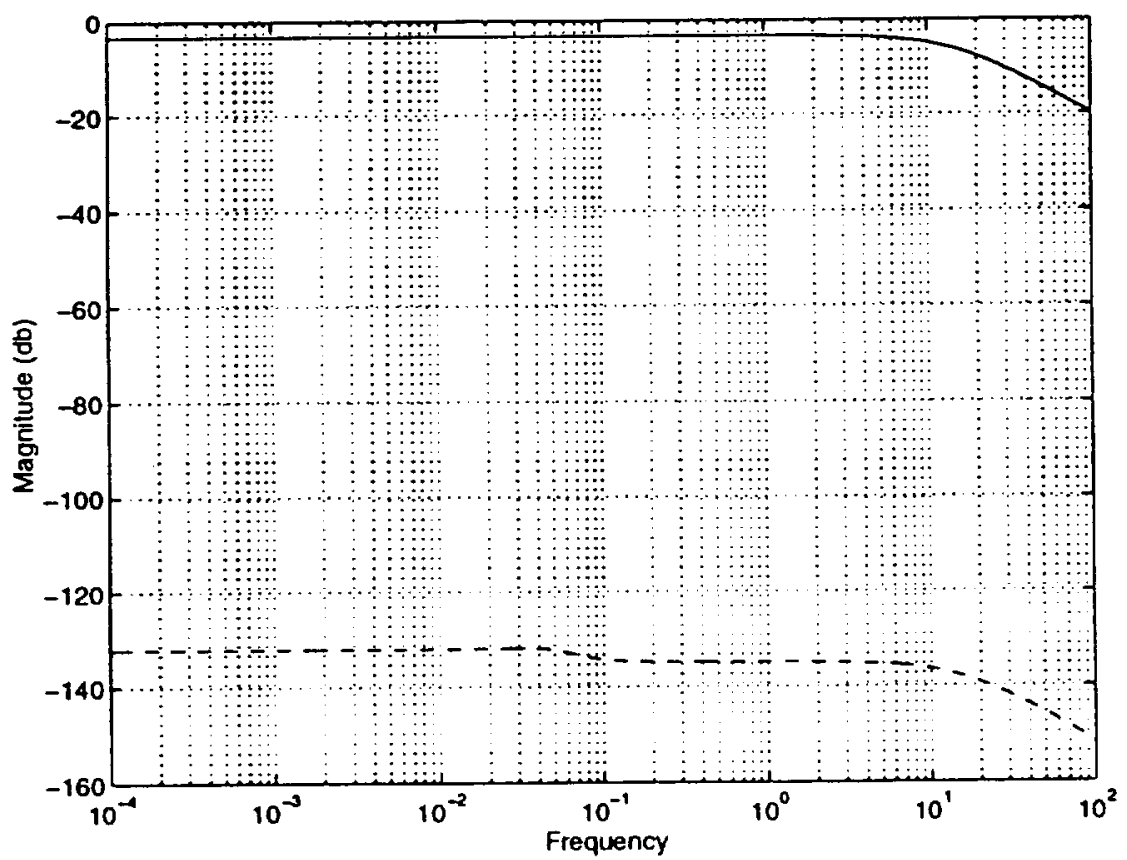

Figure 1: Frequency Response of Accerometer Fault. vs. Frequency Response of Wind Gust. (solid line - output due to $\mu_{A_{1}}$; dashed line - output. due to $\mu_{\text {wy }}$ )

Figure 1 is a plot. of the Euclidean Norms of the signal $z_{1}:=\hat{H}_{1} C(y-C \dot{r})$ due to the $\mu_{A}$, and $\mu_{w g}$. The pertinent t.ransfer functions are: 


$$
\begin{gathered}
\frac{z_{1}(s)}{\mu_{A_{2}}(s)}=\hat{H}_{1} C(s I-A+L C)^{-1} L E_{a z} \\
\frac{z_{1}(s)}{\mu_{w g}(s)}=\hat{H}_{1} C(s I-A+L C)^{-1} \hat{F}_{1}
\end{gathered}
$$

It. can been seen that at least $130 \mathrm{~dB}$ of separation exists between the output due to the wind gust and the output due to the accelerometer fault. This should be satisfactory.

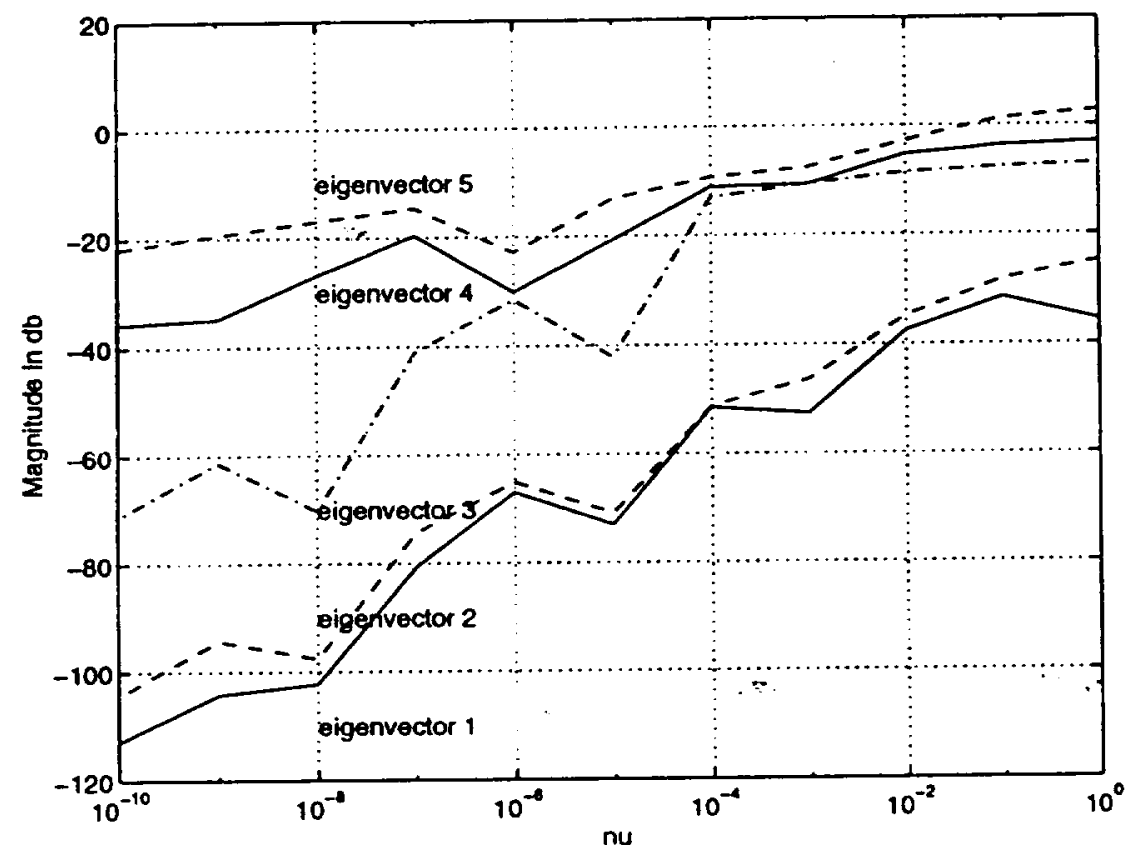

Figure 2: Inner Product of the Closed-Loop Eigenvectors and Fault Direction 1

The asymptotic convergence of the game theoretic filter to a fault detection filter structure can be demonstrated with the analysis of [4]. In that filter, the closed-loop eigenvectors are placed in such a way that for each fault $j$ its complementary detection space, $\dot{\mathcal{F}}_{i}=\sum_{j \neq i} \mathcal{F}_{j}$ is decimated. The complementary detection space is spanned by the faults which are not meant to be seen. This condition can be represented by the linear ecultion:

$$
\left[\begin{array}{cc}
A^{T}-\lambda_{i} I & C^{T} \\
\bar{F}^{T} & 0
\end{array}\right]\left[\begin{array}{c}
V_{i} \\
W_{i}
\end{array}\right]=\left[\begin{array}{l}
0 \\
0
\end{array}\right]
$$

From (126) we conchude that:

$$
\begin{aligned}
\left(A^{T}-\lambda_{i} I\right) V_{i}+C^{T} W_{i} & =0 \\
\dot{F}_{i}^{T} V_{i} & =0
\end{aligned}
$$




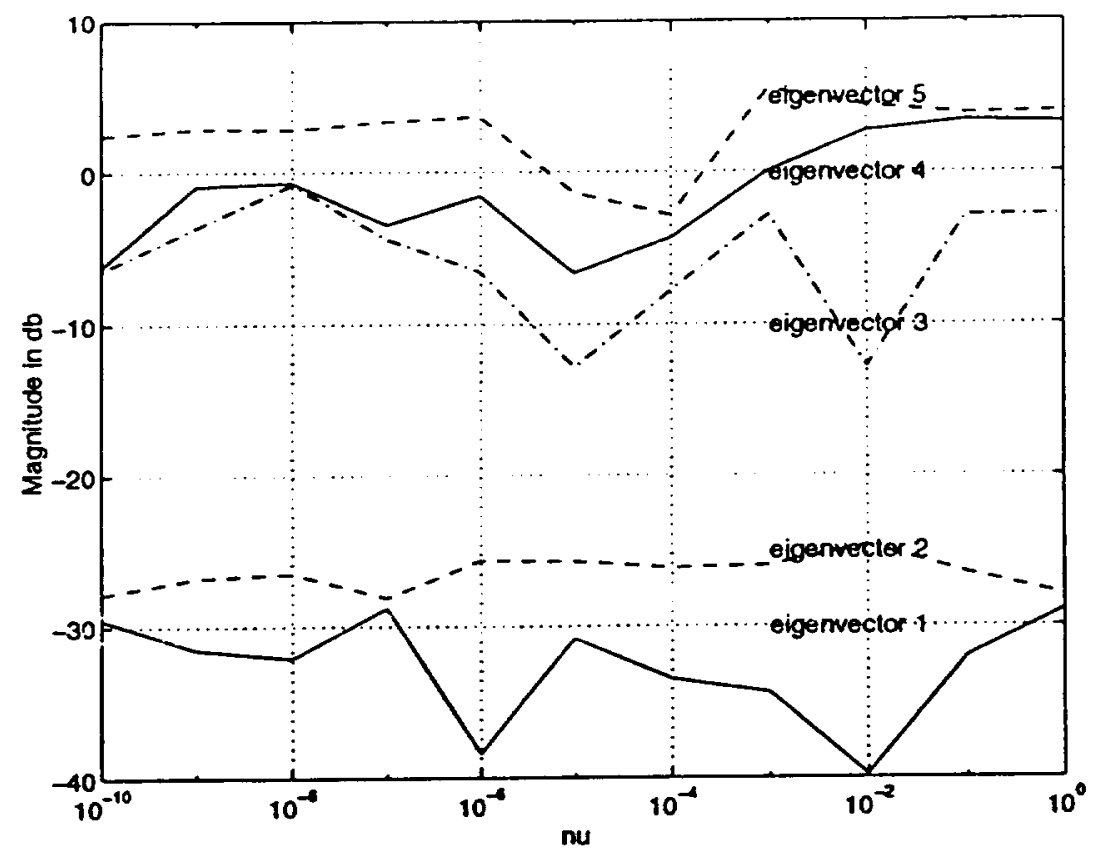

Figure 3: Inner Product of the Closed-Loop Eigenvectors and Fault Direction 2

The robust fault detection filier as described in [5] uses a result from [23] that. $W_{i}$ and $V_{i}$ are related through a gain matrix:

$$
W_{i}=L^{T} V_{i}
$$

leading to the following conditions:

$$
\begin{aligned}
\left(A^{T}+C^{T} L^{T}-\lambda_{i} I\right) V_{i} & =0 \\
\hat{F}_{i}^{T} V_{i} & =0 .
\end{aligned}
$$

We can conclude from (129) and (130), therefore, that in a fault detection filter the complementary fault directions are perpendicular to the closed-loop eigenvectors.

In Figures 2 and 3 we employ Equation 130 and examine the eigenstructure of the game theoretic filter as the parameters $\nu$ and $\gamma$ are taken to zero $(\gamma=\nu / 2)$. Figure 2 shows a plot of $F_{u g}^{\tau} V_{i}(i=1 \ldots 5)$ where $V_{i}^{\prime}$ are the eigenvectors of $\left(A+P C^{T} V^{-1} C\right)$. Figure 2 shows that the closed-loop eigenvectors (two in particular) become perpendicular to the directions of $F_{n, g}$. Since we have designed the filter to block out the transmission of $\mu_{n g}$ to a portion of the state-space, this is the result. we wondd expect.

Figure 3 shows that the closed-loop eigenvectors do not align in directions normal to the space spanned by $F_{A_{t}}$. Decreasing values of $\gamma$ seem to have no effect. on alignment, in fact. Since this is the fault that we want to detect, 
this is the result that we wallt to sees.

\subsection{Reduced-Order Filter Design via the Gol Riccati Equations}

We now repeat the example, but now we will design a lower order detection filter using the Goh Riccati Equations. The first step is to derive the transformation matrix, $\Gamma$. Since the transformation is determined via the null space of the full-order Riccati Matrix, the design process begins by finding the solution to the full-order Goh Riccati Equation (74). Because $C \hat{F}_{1}=C B_{1}=0$, we need to go to a second iteration $(i=2)$, in which we find that:

$$
B_{2}=A B_{1}=A \hat{F}_{1}
$$

Using (131) and the same weightings as in the full-order design, we find that the solution to the Goi Riccati Equation (74) is:

$$
S=\left[\begin{array}{ccccc}
-4.6032 & 7.0275 & -6.0662 & -123.5565 & -0.2697 \\
-5.2351 & 2.5842 & -2.2990 & -48.2081 & -0.1353 \\
4.4622 & -2.0830 & 1.8578 & 39.0486 & 0.1115 \\
85.2718 & -36.2789 & 32.4847 & 698.8667 & 2.0423 \\
-0.0098 & 0.0079 & -0.0069 & -0.1432 & -0.0003
\end{array}\right]
$$

Using the $Q R$ decompostion we find olttain a transformation matrix:

$$
\Gamma^{T}=\left[\begin{array}{ccccc}
-0.0537 & -0.9954 & -0.0763 & -0.0175 & 0.0091 \\
-0.0611 & -0.0667 & 0.7407 & 0.5738 & -0.3374 \\
0.0521 & 0.0332 & -0.6631 & 0.6384 & -0.3860 \\
0.9953 & -0.0596 & 0.0761 & 0.0009 & 0.0001 \\
-0.0001 & -0.0007 & -0.0062 & 0.5128 & 0.8585
\end{array}\right]
$$

Using this transformation, we reduce our state-space to a third-order system (i.e. we find the matrices $A_{11}, C_{1}$ etc.). From here we employ the reduced-order system matrices in the reduced order Goh Riccati Equation, (110). The soltution to (110) using (133) is:

$$
\bar{S}=\left[\begin{array}{ccc}
219.8389 & -14.6664 & -0.2469 \\
-14.6664 & 5.2528 & 0.1179 \\
-0.2469 & 0.1179 & 0.0035
\end{array}\right]
$$

with a corresponding gain:

$$
L=\left[\begin{array}{cccc}
-0.0066 & 0.0061 & 0.0005 & 0.0033 \\
-0.3211 & 0.1887 & 0.0068 & 0.1483 \\
8.4710 & -7.2336 & 4.3527 & -5.6715
\end{array}\right]
$$

The closed-loop eigenvalues are $-0.0375 \pm 0.0128 j,-5.1698$. Compare this to the eigenvalues of the full-order filter which are: $-0.0344 \pm 0.0448 j,-88.40 \pm 88.57 j,-14.9142$. To demonstrate the effectiveness of the reduced-order filter a linear simulation of the system was run for two cases: one with a accelerometer fault input (modelled as a step) the other with a windgust input (also a step). Figures 5 and 4 , shows that the reduced-order filter responds to the accelerometer fault input. and is relatively insensitive to the wind gust input. 


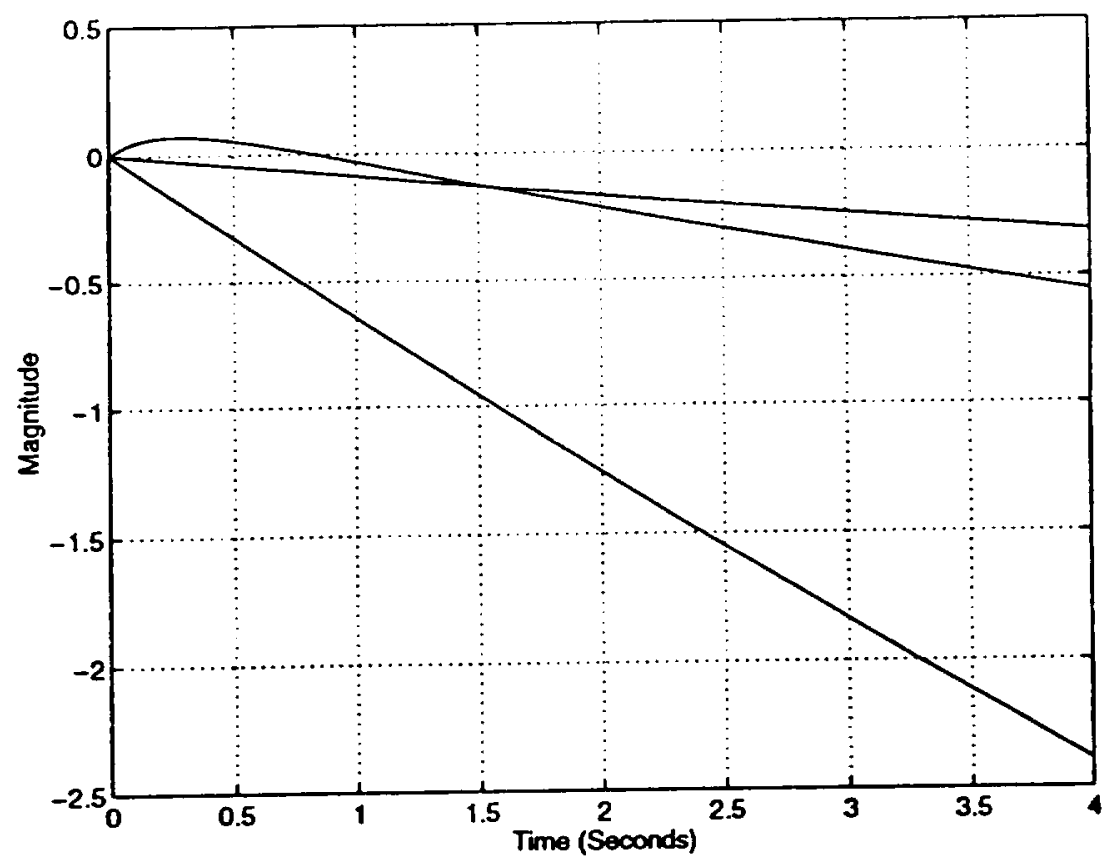

Figure 4: Reduced-Order Goh Filter Residual due to step in $\mu_{\Lambda_{4}}$ (fault to be detected)

\subsection{Reduced-Order Filter Desigu via the Lirear Matrix Inequality}

We will now demonstrate yet another design algorithm. Compared to the previous design, this approach requires more work and judgement from the engineer, but the final filter design is very effective and in some cases the approach may be numerically more reliable.

The linear matrix ineculuality (56) was solved in the steady-state case using software for semi-definite programming software ${ }^{3}$. For $\gamma=0$ and the same weighting matrices as the full-order design, it. was found that:

$$
\Pi=\left[\begin{array}{ccccc}
3.4282 & -0.1842 & 0.2482 & 6.4511 & -0.0000 \\
-0.1842 & 1.0263 & -0.9014 & -17.1934 & -0.0000 \\
0.2482 & -0.9014 & 0.7978 & 15.1839 & 0.0000 \\
6.4511 & -17.1934 & 15.1839 & 301.6193 & -0.0000 \\
-0.0000 & -0.0000 & 0.0000 & -0.0000 & 0.0000
\end{array}\right]
$$

Using the eigenvectors of the Riccati matrix (136), we obtain the following as a transformation matrix:

$$
T=\left[\begin{array}{ccccc}
0.0211 & 0.0002 & 0.0575 & 0.9979 & -0.0215 \\
-0.6823 & -0.0058 & -0.7266 & 0.0575 & 0.0568 \\
-0.7307 & -0.0066 & 0.6804 & -0.0249 & -0.0502 \\
-0.0026 & 0.0000 & -0.0769 & -0.0170 & -0.9969 \\
-0.0088 & 1.0000 & 0.0003 & -0.0000 & 0.0000
\end{array}\right]
$$

Following the results of the previons section, we choose the reduced-order estimator to be 3rd order. with the corresponding reduced Riccati Matrix:

${ }^{3}$ "SP" developed at Stanford University lyy Stephen Buyd and his students 


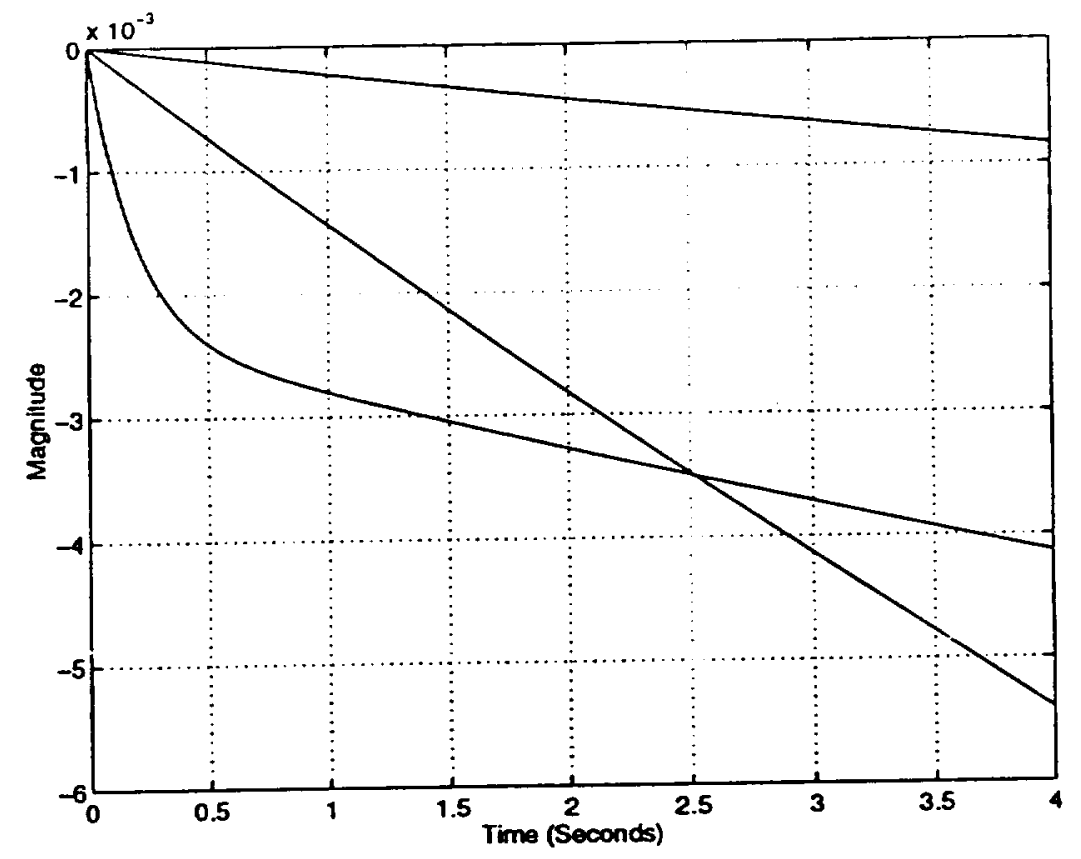

Figure 5: Reduced-Order Goh Filter Residual due to step in $\mu_{w s}$ (nuisance fault)

$$
\bar{\Pi}=\left[\begin{array}{ccc}
3.3129 & -0.0000 & 0.0000 \\
-0.0000 & 305.3302 & -0.0000 \\
0.0000 & -0.0000 & 0.0633
\end{array}\right]
$$

The resulting gain is:

$$
L=\left[\begin{array}{cccc}
0.0162 & -0.0035 & -0.0153 & -0.0005 \\
-0.6843 & 0.0740 & 0.6642 & 0.0130 \\
178.7185 & -19.9610 & -169.0275 & -5.8971
\end{array}\right]
$$

The closed-loop poles are $-0.0347 \pm 0.0448 j,-243.4$ (Note that the dominant. second-order pair of the full-order filter has been retained).

Again by simulating the reduced-order system, we find in Figures 7 and 6 that the reduced-order filter works effectively as a fault detector for an accelerometer fault in the presence of a windgust fault. Figure 6 shows that the reduced-order estimator is sensitive to $\mu_{A_{s}}$, the accelerometer fault. Whereas Figure 7 shows quite clearly that the reduced-order filter is unaffected by the presence of a windgust. fault (the residuals are on the order of $10^{-6}$ ).

\section{Conclusions}

By solving the fault detection problem via disturbance attenuation, we obtain a rame theoretic filter that bounds the transmission of disturhances and nuisance fanlts. By going to the limit of this solution, we get a fault detection filter which in the time-invariant case is eguivalent to the Beard-Jones Fault. Detection Filter. That is, the presence 


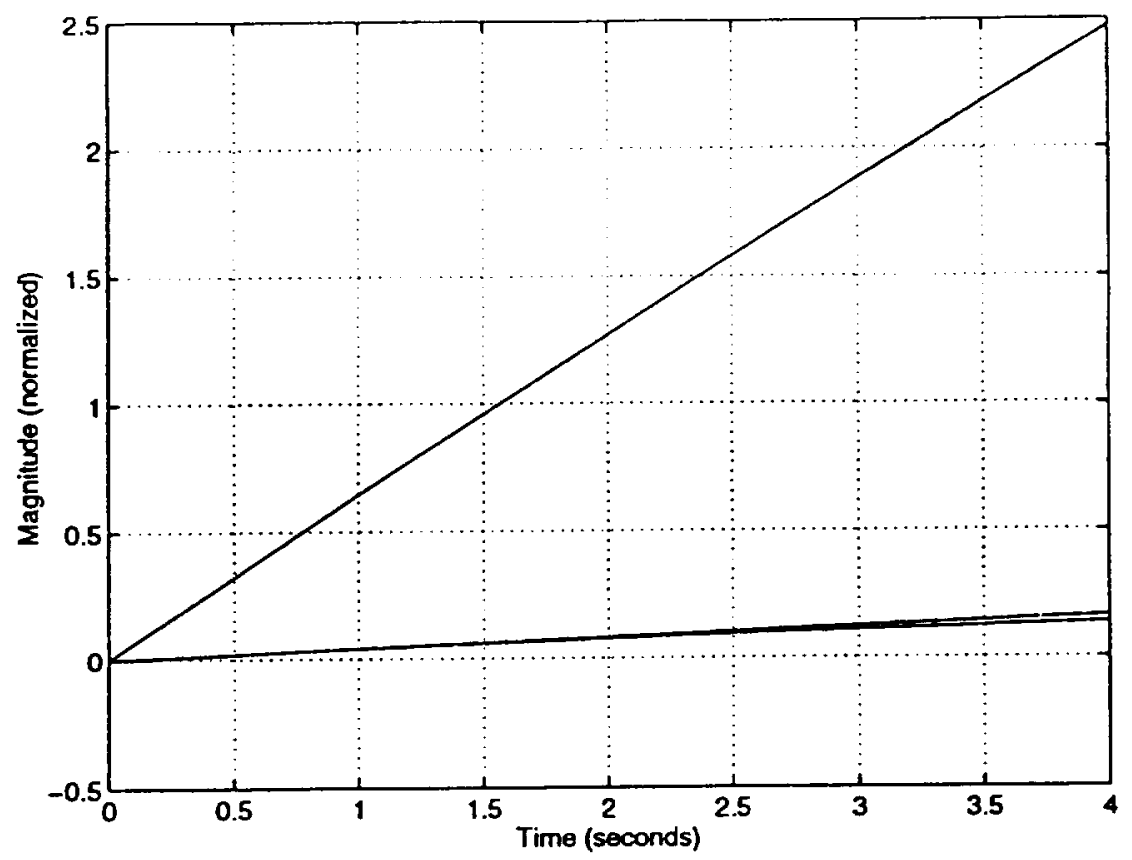

Figure 6: Reduced-Order Filter Residual due to step in $\mu_{A_{2}}$ (fault to be detected)

of the nuisance faults is iestricted to an invariant subspace that can be made unobservable through a projection. This unobservable subspace can be factored out. of total space to get a lower-order system which is uninfluenced by the nuisance faults. The same factoring process can then be applied to the game filter to get a reduced-order fault detector for the newly reduced state-space. Extensions of this latter result exist for the time-varying case, though the computation involved may be intensive.

The game theoretic approach to fatilt detection filter design is more flexible and applicable than current design methods. The designer can choose the degree to which the game filter possesses the structure of the Beard-Jones filter. This allows him to make tradeoffs between nuisance fault blocking and sensor noise rejection. The linear quadratic game used to solve the disturbance attenuation problem admits time-varying systems and can be used to incorporate parameter uncertainty into the filter design. Recent extensions of robust control such as designs which constrain pole-placement, and designs with inultiple objectives (e.g. the so-called mixed $H_{2} / H_{\infty}$ problems) suggrest that the same can be done here. The latter is of particular interest since it appears to be a logical way to detect and identify multiple faults with a single game theoretic filter.

Finally, we have shown that the limiting form of the game filter is a singular filter. Since any disturbance at.tenuation problem can be solved in the same mamner as this one, it is likely that this result applies to all such problems. That is, the limiting form of a disturbance at.tenuation problem is a singular optimization problem. This 


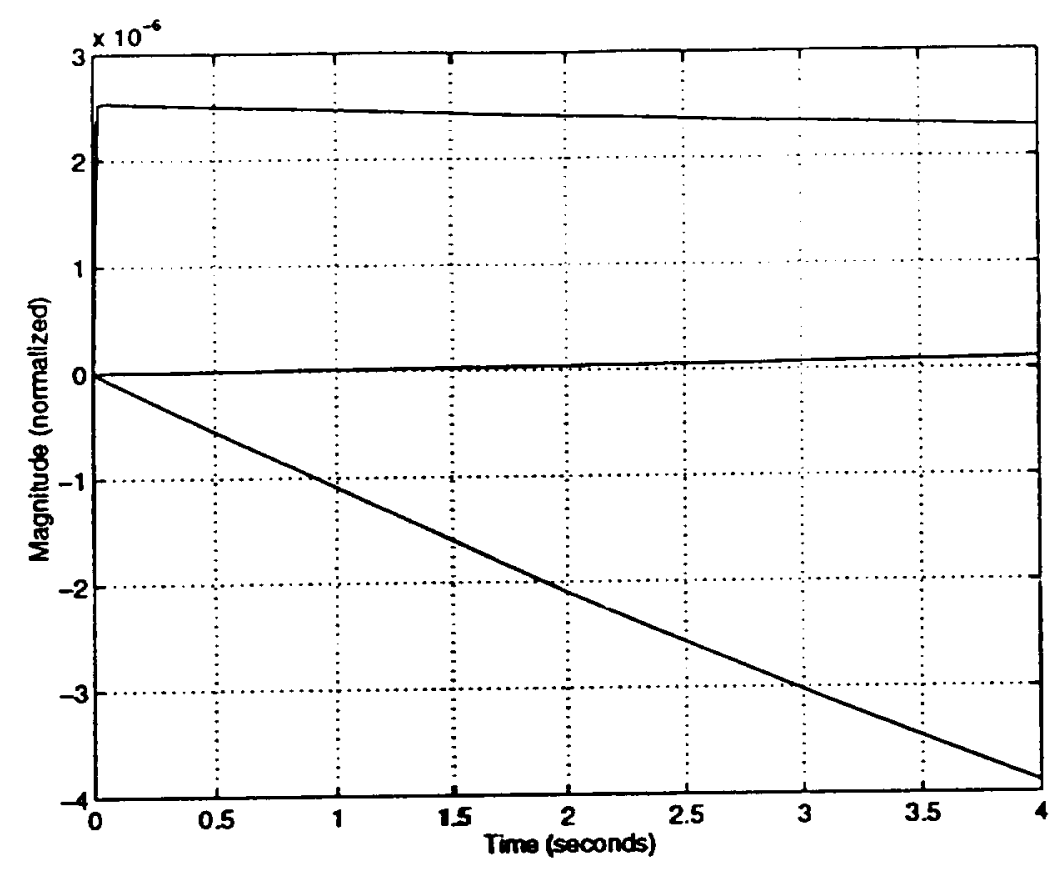

Figure 7: Reduced-Order Filter Residual due to step in $\mu_{w g}$ (nuisance fault)

makes applicable a wealth of results from singular control and it provides a new way to understand $H_{\infty}$ problems by looking at them as "almost" singular optimal control problems.

\section{References}

[1] J. E. White and J. L. Speyer, "Detection filter design: Spectral theory and algorithms," IEEE Transactions on Automatic Control, vol. AC-32, pp. 593-603, Jaly 1987.

[2] M.-A. Massoumnia, "A geometric approach to the synthesis of failure detection filters," IEEE Transactions on Automatic Control, vol. AC-31, pr. 839-846, September 1986.

[3] M.-A. Massoumnia, G. C. Verghese, and A. S. Willsky, "Fault detection and identification," IEEE Transactions on Automatic Control, vol. AC-34, pp. 316-321, March 1989.

[4] R. K. Douglas, Robust Detection Filter Design. PhD thesis, University of Texas at Austin, 1993.

[5] R. K. Douglas and J. L. Speyer, "Robust fault detection filter design," in Proceedings of the American Controls Conference, 1995.

[6] R. K. Douglas and J. L. Speyer, "An $\mathcal{H}_{\infty}$ bounded fault detection filter," in Proccedings of the American Controls Conference, 1995.

[7] G. H. Lee, Least-Squarcs and Minimax Methods for Filtering, Identification, and Detection. PhD thesis, University of California, Los Angeles, 1995.

[8] D. F. Chichka and J. L. Speyer, "An adaptive controller hased on disturbance attenuation," IEEE Transactions on Automatic Control, vol. AC-40, pp. 1220-1233, July 1995. 
[9] R. S. Mangoubi, B. D. Applely, and G. C. Verghese, "Stochastic interpretation of $\mathcal{H}_{\infty}$ and robust estimation," in Proceendings of the 93rd Conference on Decision and Control, pp. 3943-3948, IEEE, December 1994.

[10] W. M. Wonham, Linear Multivariable Control: A Geometric Approach. Springer-Verlag, second ed., 1985.

[11] D. H. Jacobson, "Totally singular quadratic optimization problems," IEEE Transactions on Automatic Control, vol. AC-16, pp. 651-658, December 1971.

[12] R. N. Banavar and J. L. Speyer, "A linear-quadratic game approach to estimation and smoothing," in Proceedings of the American Control Conference, pp. 2818-2822, 1991.

[13] I. Rhee and J. L. Speyer, "A game theoretic approach to a finite-time disturbance attenuation problem," IEEE Transactious of Automatic Control, vol. AC-36, pp. 1021-1032, September 1991.

[14] J. C. Doyle, K. Glover, P. P. Khargonekar, and B. A. Francis, "State-space soiutions to standard $\mathcal{H}_{2}$ and $\mathcal{H}_{\infty}$ control problems," IEEE Transactions on Automatic Control, vol. AC-34, pp. 831-847, August 1989.

[15] H. Kwakernaak and R. Sivan, "The maximally achievable accuracy of linear optimal regulators and linear optimal filters," IEEE Transactions on Automatic Control, vol. AC-17, pp. 79-86, February 1972.

[16] D. J. Bell and D. H. Jacobsen, Singular Optimal Control. Academic Press, 1973.

[17] D. J. Clements and B. D. Anderson, Singular Optimal Control: The Linear-Quadratic Problem, vol. 5 of Lecture Notes in Control and Information Sciences. Springer-Verlag, 1978.

[18] J. C. Schumacher, "The role of the dissapation matrix in singular optimal control," System and Control Letters, vol. 2 , no. 3 , pp. 262-266, 1983.

[19]. P. Moylan and J. Moore, "Generalizations of singular optimal control theory," Automätica, vol. 7, pp. 591-598, 1971.

[20] A. MacFarlane and N. Karcanias, "Poles and zeros of linear multivariable systems: A survey of the algebraic, geometric. and complex-variable theory," International Journal of Control, vol. 24, no. 1, pp. 33-74, 1976.

[21] W. L. Brogan, Modern Control Theory. Prentice-Hall, 3rd ed., 1991.

[22] Y. Oshman and I. Y. Bar-Itzhack, "Eigenfactor solution of the matrix riccati equation - a continuous square root algorithm," IEEE Transactions on Automatic Control, vol. AC-30, pp. 971-978, October 1985.

[23] B. C. Moore and A. J. Laub, "Computation of supremal (A, B)-invariant and controllability subspaces," IEEE Transactions on Automatic Control, vol. AC-23, October 1978. 
